\title{
An Analytical Constant Modulus Algorithm
}

\author{
Alle-Jan van der Veen (Member, IEEE) and Arogyaswami Paulraj (Fellow, IEEE)
}

\begin{abstract}
Iterative constant modulus algorithms such as Godard and CMA have been used to blindly separate a superposition of co-channel constant modulus (CM) signals impinging on an antenna array. These algorithms have certain deficiencies in the context of convergence to local minima and the retrieval of all individual CM signals that are present in the channel. In this paper, we show that the underlying constant modulus factorization problem is, in fact, a generalized eigenvalue problem, and may be solved via a simultaneous diagonalization of a set of matrices. With this new, analytical approach, it is possible to detect the number of CM signals present in the channel, and to retrieve all of them exactly, rejecting other, non-CM signals. Only a modest amount of samples are required. The algorithm is robust in the presence of noise, and is tested on measured data, collected from an experimental set-up.
\end{abstract}

\section{INTRODUCTION}

\section{A. Blind signal separation}

An elementary problem in the area of spatial signal processing is that of blind beamforming. This problem arises e.g. in the following wireless communications scenario, illustrated in figure 1. Consider a number of sources ("users") at distinct locations, all broadcasting signals at the same frequency and at the same time. The signals are received by a central platform containing an array of antennas. By linearly combining the antenna outputs, the objective is to separate the signals and to "copy" each of them without interference from the other signals. The task of the blind beamformer is to compute the proper weight vectors $\mathbf{w}_{i}$ from the measured data only, without detailed knowledge of the signals and the channel.

Mathematically, the situation is described by the simple and well-known data model

$$
X=A S,
$$

where the matrix $X: m \times n$ is a collection of $n$ snapshots from each of the $m$ antennas, $A: m \times d$ is the array response matrix, and $S: d \times n$ is the signal matrix, with $d$ rows $\mathbf{s}_{i}(i=1, \cdots, d)$ corresponding to each of the $d$ source signals. This model is a reasonably accurate description for stationary propagation environments in which the multipath has only a short delay spread (as compared to the inverse of the signal bandwidths), so that no equalization is required. The beamforming problem may thus be formulated as a structured matrix factorization problem: given $X$, find factors $A$ and $S$ satisfying certain structural properties. Once $A$ is known, the weight vectors $\mathbf{w}_{i}$ for signal copy are given by the rows of $W$, where $W=A^{\dagger}$ is the pseudo-inverse of $A$.

Although we will be concerned with blind beamforming, it is useful to note that quite similar structured factorization problems

Manuscript submitted to IEEE Trans. Signal Processing, June 1994, revised June 1995. Portions of this paper were presented at SPIE'94 [1]. A.J. van der Veen was with Dept. Computer Science/SCCM, Stanford University, Stanford, CA 94305. He is presently with the Department of Electrical Engineering/DIMES, Delft University of Technology, Delft, The Netherlands. A. Paulraj is with Information Systems Laboratory, Stanford University, Stanford, CA 94305. This research was supported by ARPA, contract no. F49620-91-C-0086, monitored by the AFOSR, and by the NSF, grant no. DMS-9205192.

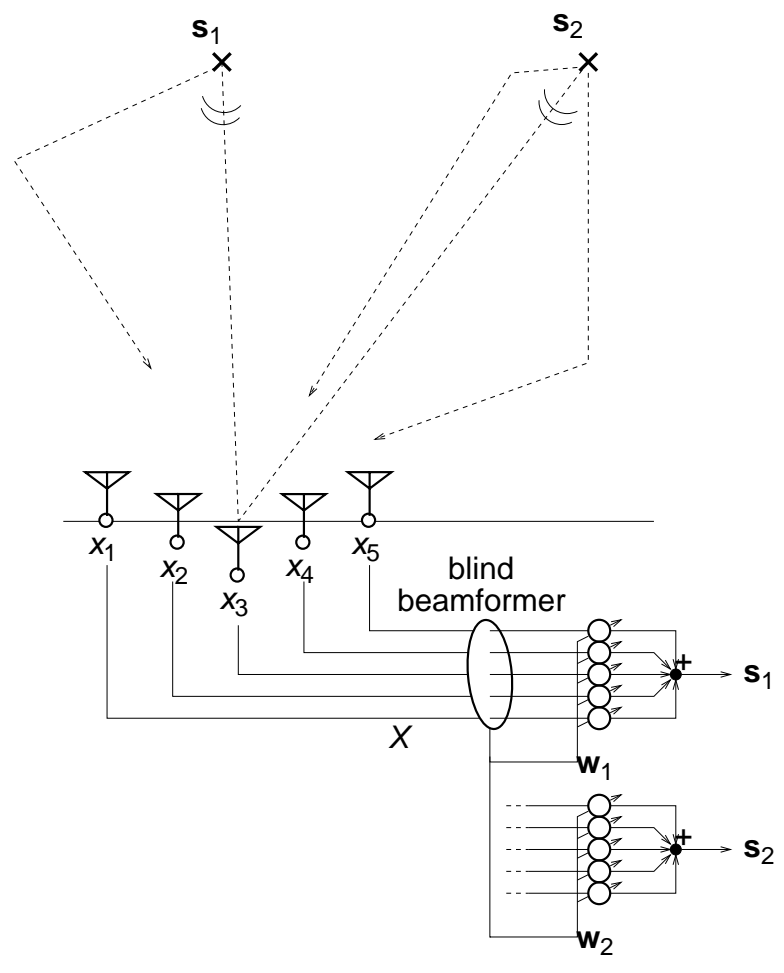

Fig. 1. Elementary blind beamforming scenario

arise in the context of blind equalization of a single source observed through an unknown time-dispersive channel. The two scenarios might even be combined into a blind multi-user separation problem in the presence of long delay multipath. Such problems are often separable into an equalization and a separation (beamforming) step (viz. e.g. [2]), so that a generic solution to the blind beamforming problem is also relevant in the combined scenario.

One mainstream of approaches for computing the structured factorization has focused on properties of the $A$-matrix. In particular, the columns of the $A$-matrix are (not always correctly) assumed to be vectors on the array manifold, each associated to a certain direction-of-arrival (DOA). The identification of the DOAs necessitates the use of calibrated antenna arrays (for the MUSIC algorithm [3]) or special array geometries (for the ESPRIT algorithm [4]), and puts serious limitations on the propagation environment as well: since in principle the direction of each multipath ray is estimated, the total number of dominant rays has to be less than the number of antennas. Moreover, rays cannot have identical delays, and diffuse multipath is not allowed. For short-delay or diffuse multipath, it might be more accurate to model each column of $A$ as the sum of two (or many) vectors on the array manifold, but then the estimation of all corresponding directions is computationally very intensive, if possible at all.

A second class of approaches, more promising in the pres- 
ence of unstructured multipath and useful in the context of blind equalization as well, exploits structural properties of the $S$-matrix that should hold and be reconstructed by the factorization. One widely used property is the constant modulus of many communication signals (e.g. FM and PM in the analog domain, and FSK, PSK, 4-QAM for digital signals). A related but distinct property is the finite alphabet of digital signals. The idea of modulus restoral has its roots in the work of Sato [5], Godard [6], and Treichler, Agee, and Larimore [7,8], all for the purpose of blind equalization; the algorithms are known as CMAs. They are usually implemented as stochastic gradient-descent optimizers of a modulus-error cost function, and are in that form quite similar to decision-directed adaptive filters or Bussgang algorithms for finite-alphabet restoral (the literature is abundant; viz. [9]). The application of the CMA to blind beamforming is straightforward and was first considered in $[10,11]$; a combined spatio-temporal CMA was proposed in [12]. Blind beamforming based solely on the finite alphabet structure is developed in [13]. Other properties of $S$ that might be used are the spectral self-coherence of communication signals, leading to the SCORE class of blind beamforming algorithms [14], and several statistical properties: e.g. the assumed independence of the sources allows to separate non-Gaussian signals based on their high-order cross-correlations [15-18].

In the context of blind equalization of digital signals, finally, the cyclostationarity of such signals may be exploited by the use of multiple antennas [19], or by sampling faster than the symbol rate and using fractionally spaced equalizers (FSE). The spatial or temporal oversampling of cyclostationary signals leads to a data matrix factorization $X=H S$ in which both the channel matrix $H$ and the signal matrix $S$ have a Hankel or Toeplitz structure. This structure by itself is already strong enough to determine the factorization, as is demonstrated in the innovative approach by Tong, Xu and Kailath [20], but perhaps more clearly visible in consecutive work $[21,22]$. It may also very effectively be combined with the properties of $S$ into a single scheme, such as FSE with CMA $[23,24]$, or FSE with finite alphabets $[2,25]$. The latter papers consider the more ambitious joint separation and equalization of multiple digital signals, but the same should be possible with FSE-CMA as well. As mentioned before, in these applications the equalization and beamforming stages are separable, and a reliable solution to the elementary blind beamforming problem is crucial.

In this paper, we will limit ourselves to blind beamforming of constant-modulus (CM) signals, assuming no other properties of the signals except their independence. This blind CM beamforming problem was introduced in $[10,11]$, and solved using the CMA [7], but in a restricted form: only the reception of a single signal-of-interest among other interfering signals was considered. It was observed that the algorithm can lock onto one of the interfering signals rather than the desired signal. In later papers, this misbehavior was used to set up a multi-stage CMA, in which the intention is to capture all incident CM signals [10,26, 27]. The output of a first CMA stage results in the detection of the first CM signal. By an orthogonal projection, or an LMSimplementation of it, this signal is subtracted from the data matrix, and the resulting filtered data is fed to a second CMA stage in order to detect a possible second CM signal. However, for short data sequences, the signals are not yet orthogonal to each other, and the projection leads to a misadjustment in the second and subsequent stages, thus limiting its performance. To mitigate this effect, the forced orthogonality of the signals may be relaxed [28-30] by only making sure that they are "sufficiently" independent of each other. In these schemes, a number of CMAs are running in parallel, all started from distinct initializations. Orthogonality is tested and weakly restored at the end of an update block of $n$ samples.

Although the latter approach has been successfully demonstrated in an on-line outdoor experiment [31], it provides only a heuristic solution to the underlying, very tough problem: how can gradient descent techniques be used to converge reliably to all minima of the cost function. Indeed, how do we know the number of minima to look for in the first place? When only a finite block of data is available, it is very likely that there are local minima of the sample cost function as well, not corresponding to any of the source signals. Depending on the initialization of the gradient descent optimization, the CMAs do converge sometimes to these solutions. In this respect, it should be noted that global convergence of the CMA has only been proven for infinite sets of data (or in the averaged sense) [32], and only for scenarios that admit a perfect solution. ${ }^{1}$ Finally, convergence of the CMAs may be slow and irregular, especially for weak sources and short data sets.

\section{B. Contributions}

In this paper, we introduce a new approach to the constant modulus factorization problem. We show that the problem is essentially a generalized eigenvalue problem and can be solved analytically, by a deterministic algorithm and using only a finite set of data ( $n$ samples of $m$ antennas). In particular,

- For $d \leq m$ sources, and without noise, $n>d^{2}$ samples are sufficient to compute $A$ and $S$ exactly, via a certain eigenvalue problem.

- For $n>d^{2}$, it is possible to detect the number of CM signals present in $X$. This implies that not all sources have to generate $\mathrm{CM}$ signals, although the algorithm only recovers the CM sources.

- With $X$ distorted by additive noise, a generalization of the algorithm is robust in finding $S$, even when $n$ is quite small. This is demonstrated with experimental data.

The algorithm is derived by setting up the equations for the weight vector $\mathbf{w}$ such that $\mathbf{w} X$ is a $\mathbf{C M}$ signal (section II). This gives $n$ quadratic equations in the entries of $\mathbf{w}$, which can be linearized when written in terms of the Kronecker product $\mathbf{w} \otimes \overline{\mathbf{w}}$, a vector with $d^{2}$ entries ( $\overline{\mathbf{w}}$ is the complex conjugate of $\left.\mathbf{w}\right)$. If $n>$ $d^{2}$, then the dimensionality of the solution space of this linear system of equations indicates how many CM signals are present in $X$. Most solution vectors of the linear system do not have the Kronecker structure $\mathbf{w} \otimes \overline{\mathbf{w}}$; the core of the CM problem is to find

\footnotetext{
${ }^{1}$ This is not so much an issue in blind beamforming where the usual assumption is that the number of sources is at most equal to the number of sensors, but has caused much confusion in the blind equalization of FIR channels by FIR equalizers, viz. [33]. Current insight is that a fractionally sampled equalizer allows a perfect solution and thus assures asymptotic global convergence [24].
} 
those that do. It is shown in section III how this problem can be transformed into a generalization of an eigenvalue problem: the simultaneous diagonalization of a number of matrices. Without noise, this problem has an essentially unique solution which can be found using standard linear algebra tools. With noise added to $X$, there is in general no exact diagonal solution, and we have to find an approximate simultaneous diagonalization. This is a challenging, non-standard linear algebra problem, for which we propose an algorithm that exhibits quadratic convergence in simulations (section IV). In section V, the algorithm is applied both to computer generated data and to measured data sets, with very good results.

It is not the first time that a Kronecker approach has been proposed to solve the CM problem, viz. e.g. [34-36]. However, these authors operate in the equalization context and try to find only one structured solution $\mathbf{w} \otimes \overline{\mathbf{w}}$ to the linear system, ignoring the fact that there might be more such solutions (in the equalization context, this occurs if the equalizer length is too long). Interestingly enough, an entirely similar simultaneous diagonalization problem did turn up in fourth-order cumulantbased techniques for blind separation of multiple non-Gaussian signals [15-18]. With hindsight, one might perhaps say that CM signals are deterministic counterparts of non-Gaussian signals, but only as far as the fourth-order cumulant is concerned. At this point, we are only aware that there must be connections, but the details remain to be sorted out.

\section{Notation}

Lower case bold (as in w) denotes either a row or a column vector. Its $i$-th entry is sometimes denoted as $(\mathbf{w})_{i} . \mathbf{w}^{T}$ is the transpose, $\overline{\mathbf{w}}$ is the complex conjugate, and $\mathbf{w}^{*}$ is the complex conjugate transpose. $\otimes$ is the Kronecker product. For matrices, $A^{\dagger}$ denotes the Moore-Penrose pseudo-inverse, $\operatorname{row}(A)$ denotes the row span (co-range) of $A$.

\section{PROBLEM FORMULATION AND TRANSFORMATION}

\section{A. Problem statement; uniqueness}

In this section, we discuss the actual problem that will be solved. Starting from the data model $X=A S$ in (1), we first note that without loss of generality, the constant modulus of all signals may be modeled to be equal to 1: any other value of the amplitude of one of the signals is absorbed in the $A$-matrix by a proper scaling of corresponding columns of $A$ and rows of $S$. Hence, the problem we consider is, for a given data matrix $X$, to find a factorization

$$
X=A S, \quad \text { with } A, S \text { full rank, }\left|S_{i j}\right|=1 .
$$

A slightly more general way to formulate the problem is obtained by premultiplying (2) with $W=A^{\dagger}$, where $A^{\dagger}$ denotes the pseudo-inverse of $A$ :

Problem $P 1$ (CM factorization problem) For a given data matrix $X: m \times n$ of rank $d$, find $\delta$ and $W: \delta \times m$, such that

$$
W X=S, \quad\left|S_{i j}\right|=1,
$$

where $S$ is of full rank and $\delta \leq d$ is as large as possible.
In this formulation, $X$ is a linear combination of $d$ signals, but only $\delta \leq d$ of them are of CM type. Only the CM signals will be reconstructed by the beamformer.

The formulations $X=A S$ and $W X=S$ are equivalent only if the factorization $X=A S$ is essentially unique, meaning that the only CM signals that can be constructed by the beamformer are the signals that were originally sent, and not some spurious "ghost" signals. Trivial transformations such as the choice of ordering of the rows of $S$ and the complex phases of the entries of the first column of $S$ cannot be avoided but lead to an admissible form of non-uniqueness. Save for these transformations, and under conditions that $A, S$ are full rank and the sources generating $S$ are "sufficiently independent" and have "sufficient phase richness", uniqueness is guaranteed with probability 1 once $n$ is "large enough". This is well known for $\delta=d, n \rightarrow \infty$, and analog CM signals (viz. e.g., [7] for equalization, [37] for beamforming). One may have concerns on the "sufficient phase richness" of digital CM signals with small constellations, but in fact even BPSK signals give unique factorizations [13]. However, a sharpening of the $n \rightarrow \infty$ condition is possible.

Lemma 1: Suppose that $X: m \times n$ has rank $d$ and that the factorization $\left\{X=A S,\left|S_{i j}\right|=1\right\}$ is unique for $n \rightarrow \infty$. Then the factorization is in general already unique for $n \geq 2 d$.

Proof: See Appendix B.

The algorithm which we derive in this paper requires $n>d^{2}$, which is still quite reasonable for small values of $d$ (say $d<10$ ).

\section{B. The Gerchberg-Saxton algorithm}

Denote by $\operatorname{row}(X)$ the subspace spanned by the rows of $X$ (the co-range of $X$ ), and define the set of CM signals as

$$
\mathcal{C M}=\left\{S|| S_{i j} \mid=1, \text { all } i, j\right\} .
$$

Problem P1 asks for all row vectors $\mathbf{w}$ (the rows of $W$ ) such that $\mathbf{w} X=\mathbf{s}$ is a CM signal, for linearly independent signals $\mathbf{s}$. Hence we have the following lemma:

Lemma 2: Problem P1 is precisely equivalent to finding all linearly independent signals $\mathbf{s}$ that satisfy

$$
\begin{aligned}
& \text { (A) } \mathbf{s} \in \operatorname{row}(X), \\
& \text { (B) } \mathbf{s} \in \mathcal{C M} .
\end{aligned}
$$

From this formulation, it is straightforward to devise an algorithm based on alternating projections: start with a (random) choice of $\mathbf{s}$ in the row span of $X$, and alternatingly project it onto $\mathcal{C M}$ and back onto the row span of $X$. The set $\mathcal{C M}$ is not a linear subspace, so that the the projection onto $\mathcal{C M}$ is non-linear:

$$
\mathbf{P}_{\mathcal{C M}}(\mathbf{y})=\left[\frac{(\mathbf{y})_{1}}{\left|(\mathbf{y})_{1}\right|}, \cdots, \frac{(\mathbf{y})_{n}}{\left|(\mathbf{y})_{n}\right|}\right]
$$

i.e., every entry of the vector is radially projected onto the complex unit circle. It is customary to estimate weight vectors $\mathbf{w}$ rather than signals, in which case the alternating projection algorithm is expressed as the iteration

$$
\mathbf{w}^{(i+1)}=\left[\mathbf{P}_{\mathcal{C M}}\left(\mathbf{w}^{(i)} X\right)\right] X^{\dagger}
$$

(Note that $\mathbf{s}^{(i)}=\mathbf{w}^{(i)} X$, and that $\cdot X^{\dagger} X$ is a projection onto the row span of $X$.) 
It is interesting to note that this is a well-established algorithm in the field of optics for solving the phase-retrieval problem, where it is known as the Gerchberg-Saxton algorithm (GSA) [38]. The connection of the phase-retrieval problem with the CM problem was made only recently [37]. Essentially the same algorithm was derived from the CMA by Agee [39], called the LSCMA, and claimed to have faster convergence than the standard CMA. It is also closely related to the OCMA variant by Gooch and Lundell [11], who replaced the LMS-type updating of the CMA by an RLS-update. One difference of the GSA and LSCMA with other CMA methods is that they are block methods: they iterate on $X$, rather than update vectors $\mathbf{x}_{k}$. Hence, they typically require less data ( $\operatorname{smaller} n)$, although of course the standard iterative CMA could reuse old data as well. Conversely, the GSA/LSCMA methods could be used on data matrices of increasing sizes, by introducing updating versions for the pseudoinverse, which leads to the OCMA. The disadvantage of using these iterative algorithms on small finite data sets is that global convergence properties are lost: spurious local minima could be introduced. It is not known how large the block-size has to be before the asymptotic global convergence results are applicable.

\section{Equivalent problem statement}

However, our intent is to compute an exact solution to the problem in lemma 2 via analysis, and not via alternating projections. Recall that we are searching for all vectors $\mathbf{s}$ that are in the row span $\operatorname{row}(X)$ and also have the CM property. The first property has so far been captured as requiring $\mathbf{s}=\mathbf{w} X$, but it is more convenient to take linear combinations of a minimal (orthonormal) basis for the row span of $X$. This avoids problems with non-uniqueness of $\mathbf{w}$ when $d<m$, and makes sure that different linear combinations lead to different signals.

Thus let $X=U \Sigma V: U \in \mathbb{C}^{m \times m}, \Sigma \in \mathbb{R}^{m \times n}, V \in \mathbb{C}^{n \times n}$ be a singular value decomposition of $X$ [40]: $U$ and $V$ are unitary matrices containing the singular vectors, and $\Sigma$ is a real diagonal matrix with non-negative entries: the singular values. Suppose that there are $d$ sources, so that the rank of $X$ is equal to $d$. Only $d$ singular values are non-zero, and we collect the corresponding right singular vectors of $V$ in a matrix $\hat{V} \in \mathbb{C}^{d \times n}$. The rows of $\hat{V}$ form an orthonormal basis of the row span of $X$, and we can rewrite condition $(A)$ in lemma 2 as

$$
(A): \quad \mathbf{s} \in \operatorname{row}(X) \Leftrightarrow \mathbf{s}=\mathbf{w} \hat{V}, \quad \hat{V}: d \times n .
$$

Here, the weight vector $\mathbf{w}$ is not precisely the same as before: it is now acting on the orthogonal basis vectors of $\operatorname{row}(X)$, rather than directly on $X$. This reduces the number of parameters to estimate from $m$ to $d$, and ensures that linearly independent $\mathbf{w}$ result in linearly independent $\mathbf{s}$. A second advantage of an orthogonal basis is that the corresponding matrix $W$ (acting on $\hat{V}$ instead of $X$ ) has a condition number that tends to 1 as $n$ grows: for uncorrelated signals and large $n, S \sqrt{n}$ becomes an isometry and $W / \sqrt{n}$, as a mapping of one isometry into another, becomes unitary. When using $X$ instead of $\hat{V}, W$ would have a bad condition number if signals come from close directions. ${ }^{2}$

\footnotetext{
${ }^{2}$ The idea to switch to an orthogonal basis and force $W / \sqrt{n}$ to be close to unitary can be used to enhance convergence to independent solutions in iterative CMA algorithms as well. Similar to Pro-ESPRIT [41], such an approach could be called a Procrustes-CMA.
}

To rewrite condition $(A): \mathbf{s} \in \mathcal{C} \mathcal{M}$, put $\hat{V}=\left[\mathbf{v}_{1} \cdots \mathbf{v}_{n}\right]$, where $\mathbf{v}_{i} \in \mathbb{C}^{d}$ is the $i$-th column in $\hat{V}$. Then

$$
\begin{aligned}
(A): & \mathbf{s}=\left[(\mathbf{s})_{1} \cdots(\mathbf{s})_{n}\right] \in \mathcal{C M} \\
& \Leftrightarrow\left[\left|(\mathbf{s})_{1}\right|^{2} \cdots\left|(\mathbf{s})_{n}\right|^{2}\right]=\left[\begin{array}{lll}
1 & \cdots & 1
\end{array}\right] \\
& \Leftrightarrow\left\{\begin{array}{c}
\mathbf{w}_{1} \mathbf{v}_{1}^{*} \mathbf{w}^{*}=1 \\
\vdots \\
\mathbf{w}_{n} \mathbf{v}_{n}^{*} \mathbf{w}^{*}=1
\end{array}\right.
\end{aligned}
$$

If we define $P_{k}=\mathbf{v}_{k} \mathbf{v}_{k}^{*} \in \mathbb{C}^{d \times d}$, for $k=1, \cdots, n$, then the above derivation has shown that problem $\mathrm{P} 1$ is precisely equivalent to finding all linearly independent vectors $\mathbf{w}$ such that

$$
\mathbf{w} P_{k} \mathbf{w}^{*}=1, \quad k=1, \cdots, n,
$$

which calls for the simultaneous solution of $n$ quadratic equations into the entries of $\mathbf{w}$, or the intersection of $n$ ellipsoids. To find all solutions, the approach is to expand these equations in the entries of $\mathbf{w}$, which leads to Kronecker products.

For matrices $Y \in \mathbb{C}^{d \times d}$ and vectors $\mathbf{y} \in \mathbb{C}^{d^{2}}$, denote

$$
\begin{gathered}
\operatorname{vec}(Y):=\left[\begin{array}{c}
Y_{11} \\
Y_{12} \\
\vdots \\
Y_{21} \\
\vdots \\
Y_{d d}
\end{array}\right], \\
\operatorname{vec}^{-1}(\mathbf{y}):=\left[\begin{array}{llll}
(\mathbf{y})_{1} & (\mathbf{y})_{2} & \cdots & (\mathbf{y})_{d} \\
(\mathbf{y})_{d+1} & (\mathbf{y})_{d+2} & \cdots & (\mathbf{y})_{2 d} \\
\vdots & & \ddots & \vdots \\
(\mathbf{y})_{d^{2}-d+1} & & & (\mathbf{y})_{d^{2}}
\end{array}\right] .
\end{gathered}
$$

If we set

$$
\begin{aligned}
& \mathbf{y}=\operatorname{vec}\left(\mathbf{w}^{*} \mathbf{w}\right)=\left[\begin{array}{c}
(\mathbf{w})_{1}(\mathbf{w})_{1}^{*} \\
(\mathbf{w})_{1}(\mathbf{w})_{2}^{*} \\
\vdots \\
(\mathbf{w})_{1}(\mathbf{w})_{d}^{*} \\
(\mathbf{w})_{2}(\mathbf{w})_{1}^{*} \\
\vdots \\
(\mathbf{w})_{d}(\mathbf{w})_{d}^{*}
\end{array}\right]=: \mathbf{w} \otimes \overline{\mathbf{w}} \in \mathbb{C}^{d^{2} \times 1} \\
& \mathbf{p}_{k}=\operatorname{vec}\left(P_{k}\right)^{T}=\left[\begin{array}{llll}
\left(P_{k}\right)_{11} & \left(P_{k}\right)_{12} & \cdots & \left(P_{k}\right)_{d d}
\end{array}\right],
\end{aligned}
$$

then the quadratic expression $\mathbf{w} P_{k} \mathbf{w}^{*}$ is "linearized" as

$$
\mathbf{w} P_{k} \mathbf{w}^{*}=\mathbf{p}_{k} \mathbf{y} .
$$

Collect the $n$ condition vectors $\mathbf{p}_{k}$ in one matrix $P$ of size $n \times d^{2}$ :

$$
P=\left[\begin{array}{c}
\mathbf{p}_{1} \\
\vdots \\
\mathbf{p}_{n}
\end{array}\right]=\left[\begin{array}{c}
{\left[\overline{\mathbf{v}}_{1} \otimes \mathbf{v}_{1}\right]^{T}} \\
\vdots \\
{\left[\overline{\mathbf{v}}_{n} \otimes \mathbf{v}_{n}\right]^{T}}
\end{array}\right]
$$

Lemma 3: Problem P1 is precisely equivalent to finding all linearly independent vectors $\mathbf{y}$ satisfying

$$
\begin{aligned}
P \mathbf{y} & =\left[\begin{array}{c}
1 \\
\vdots \\
1
\end{array}\right] \\
\mathbf{y} & =\mathbf{w} \otimes \overline{\mathbf{w}}
\end{aligned}
$$


For each solution $\mathbf{w}$, the corresponding CM signal is given by $\mathbf{s}=\mathbf{w} \hat{V}$.

Proof: To prove that this problem is equivalent to solving equation (4), and hence problem $\mathrm{P} 1$, it remains to show that a set of solutions $\left\{\mathbf{w}_{k} \otimes \overline{\mathbf{w}}_{k}\right\}_{1}^{\delta}$ is linearly independent if and only if the corresponding set $\left\{\mathbf{w}_{k}\right\}_{1}^{\delta}$ is linearly independent. This is straightforward; see Appendix B.

The linear system of equations (9) is overdetermined for $n>$ $d^{2}$. Nonetheless, if there is more than one CM signal present in $X$, there has to be more than one solution $\mathbf{y}$ to the linear system, and because they are linearly independent, $P$ has to be singular. Hence, the set of independent solutions $\mathbf{y}$ to the linear system is not unique: any vector in the kernel of $P$ can be added to any solution $\mathbf{y}$. The second condition (10) restricts the solution space to vectors $\mathbf{y}$ that have a certain structure: they must be expressible as a Kronecker product of a vector with its complex conjugate. Note that it is not sufficient to compute solutions $\mathbf{y}$ to the system of equations and hope that they have the required structure $\mathbf{y}=\mathbf{w} \otimes \overline{\mathbf{w}}$.

In general, the solution space to (9) can be written as an affine space of the form $\mathbf{y}=\mathbf{y}_{0}+\alpha_{1} \mathbf{y}_{1}+\cdots+\alpha_{\ell} \mathbf{y}_{\ell}$, where $\mathbf{y}_{0}$ is a particular solution to (9), and $\mathbf{y}_{1}, \cdots, \mathbf{y}_{\ell}$ is a basis of the kernel of $P$. We find it more convenient to work with a fully linear solution space, which is obtained via a linear transformation, as follows. Let $Q$ be any $n \times n$ unitary matrix such that

$$
Q\left[\begin{array}{c}
1 \\
\vdots \\
1
\end{array}\right]=\left[\begin{array}{c}
n^{1 / 2} \\
0 \\
\vdots \\
0
\end{array}\right]
$$

Simple choices for $Q$ suffice; e.g., $Q$ could be a Householder transformation [40],

$$
Q=I-2 \frac{\mathbf{q q}^{*}}{\mathbf{q}^{*} \mathbf{q}}, \quad \mathbf{q}=\left[\begin{array}{c}
1 \\
1 \\
\vdots \\
1
\end{array}\right]-\left[\begin{array}{c}
n^{1 / 2} \\
0 \\
\vdots \\
0
\end{array}\right],
$$

or $Q$ could be a DFT (discrete Fourier transform) matrix. Apply $Q$ to $P$ :

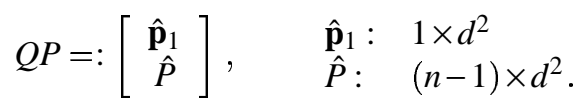

With these definitions, we have

$$
P \mathbf{y}=\left[\begin{array}{c}
1 \\
\vdots \\
\dot{1}
\end{array}\right] \quad \Leftrightarrow \quad\left\{\begin{array}{lll}
(i) & \hat{\mathbf{p}}_{1} \mathbf{y}= & n^{1 / 2} \\
(i i) & \hat{P} \mathbf{y}= & 0 .
\end{array}\right.
$$

We will show that the first condition can always be satisfied by scaling a non-trivial solution to the second equation. This then leads to the following equivalent problem statement:

Lemma 4: The CM problem $\mathrm{P} 1$ is precisely equivalent to the following problem. Let $X$ be a given matrix. With $\hat{P} \in \mathbb{C}^{(n-1) \times d^{2}}$ and $\hat{V}$ constructed from $X$, find all linearly independent non-zero solutions $\mathbf{y}$ that satisfy

$$
\begin{aligned}
\hat{P} \mathbf{y} & =0 \\
\mathbf{y} & =\mathbf{w} \otimes \overline{\mathbf{w}} .
\end{aligned}
$$

For each solution $\mathbf{w}$, scaled such that $\|\mathbf{w}\|=n^{1 / 2}$, the vector $\mathbf{s}=\mathbf{w} \hat{V}$ is a CM signal contained in $X$.

Proof: See Appendix B.

Suppose that the dimension of the kernel of $\hat{P}$ is equal to some number $\hat{\delta}$ (we will argue in section III-A below that in general $\hat{\delta}$ is, indeed, equal to the number of CM signals). Let $\left\{\mathbf{y}_{1}, \cdots, \mathbf{y}_{\hat{\delta}}\right\}$ be a basis for this kernel. It can be computed via a $\mathrm{QR}$ factorization, or, with more numerical accuracy, from an SVD of $\hat{P}$. In the latter case, $\left\{\mathbf{y}_{k}\right\}_{1}^{\delta}$ are the right singular vectors corresponding to the $\delta$ singular values of $\hat{P}$ that are zero. With a basis of the kernel, any solution $\mathbf{y}$ to $\hat{P} \mathbf{y}=0$ can be written as $\mathbf{y}=\alpha_{1} \mathbf{y}_{1}+\cdots+\alpha_{\hat{\delta}} \mathbf{y}_{\hat{\delta}}$, for arbitrary coefficients $\alpha_{i} \in \mathbb{C}$. The condition that $\mathbf{y}=\mathbf{w} \otimes \overline{\mathbf{w}}$ as well is more conveniently expressed as $Y=\mathbf{w}^{*} \mathbf{w}$, where $Y=$ $\operatorname{vec}^{-1}(\mathbf{y})$. Likewise, denote by $Y_{k}=\operatorname{vec}^{-1}\left(\mathbf{y}_{k}\right), k=1, \cdots, \hat{\delta}$, the corresponding $d \times d$ matrices constructed from the chosen basis of $\operatorname{ker}(\hat{P})$. Since

$$
\begin{aligned}
& \alpha_{1} \mathbf{y}_{1}+\cdots+\alpha_{\hat{\delta}} \mathbf{y}_{\hat{\delta}}=\mathbf{w} \otimes \overline{\mathbf{w}} \\
& \Leftrightarrow \quad \alpha_{1} Y_{1}+\cdots+\alpha_{\hat{\delta}} Y_{\hat{\delta}}=\mathbf{w}^{*} \mathbf{w}
\end{aligned}
$$

it is seen that, in essence, we have to find scalar linear combinations of a set of matrices (a generalized matrix pencil for more than two matrices) such that the result is a rank 1 hermitian matrix, hence factorizable as $\mathbf{w}^{*} \mathbf{w}$. Linearly independent solutions $\mathbf{w}$ correspond to linearly independent $\mathbf{y}$, and in turn to linearly independent parameter vectors $\left[\alpha_{1} \cdots \alpha_{\hat{\delta}}\right.$. Hence, we may rewrite the conditions (13) in terms of the $Y_{k}$, which gives a new problem statement that is entirely equivalent to the original CM problem.

Problem P2 (Equivalent CM problem) Let $X$ be the given matrix, from which the set of $d \times d$ matrices $\left\{Y_{1}, \cdots, Y_{\hat{\delta}}\right\}$ are derived as discussed above. The CM problem $\mathrm{P} 1$ is precisely equivalent to the following problem: determine all independent non-zero parameter vectors $\left[\alpha_{1} \cdots \alpha_{\hat{\delta}}\right]$ such that

$$
\alpha_{1} Y_{1}+\cdots+\alpha_{\hat{\delta}} Y_{\hat{\delta}}=\mathbf{w}^{*} \mathbf{w}
$$

For each solution $\mathbf{w}$, scaled to $\|\mathbf{w}\|=n^{1 / 2}$, the vector $\mathbf{s}=\mathbf{w} \hat{V}$ is a CM signal contained in $X$.

Finding all solutions to (14) is thus the core of the CM problem. In the next section, we show that (14) is, in fact, a generalized eigenvalue problem.

\section{Remark}

The given definition for the vec-operation in (5)-(6) does not make use of the fact that we only apply it to hermitian matrices $\mathbf{w}^{*} \mathbf{w}$ and $P_{k}$. Other choices that do make use of this are possible; e.g., we could define vec $\left(\mathbf{w}^{*} \mathbf{w}\right)$ to be a real vector containing $\operatorname{Re}\left((\mathbf{w})_{i}(\mathbf{w})_{j}^{*}\right), \operatorname{Im}\left((\mathbf{w})_{i}(\mathbf{w})_{j}^{*}\right)$ instead of the redundant $(\mathbf{w})_{i}(\mathbf{w})_{j}^{*}$ and $(\mathbf{w})_{j}(\mathbf{w})_{i}^{*}$. This transformation to real vectors leads to an equivalent but numerically and computationally favorable variant of the procedure and is detailed in Appendix A. The result is that, with this alternate definition, $P$ and $\hat{P}$ are transformed to real matrices, and that the basis matrices $Y_{k}$ constructed from the kernel of $\hat{P}$ are hermitian by construction. The coefficients $\alpha_{k}$ to be computed may then be restricted to reals as well.

\section{EXACT SOLUTION TO PROBLEM P2}

In this section, we will show that problem $\mathrm{P} 2$ admits an exact solution, which can be computed using standard linear algebra 
techniques. We still operate under the assumption that there exists a solution, and that the solution is essentially unique. For computability, we will now also require that $n>d^{2}$.

\section{A. Estimation of the number of CM signals}

We first show that there is a relation between $\delta$, the number of $\mathrm{CM}$ signals that are present in $X$, and $\hat{\delta}$, the dimension of the kernel of $\hat{P}$. With $\delta$ independent constant modulus signals, there are $\delta$ linearly independent solutions $\mathbf{w}$ to the $\mathrm{CM}$ factorization problem, corresponding to $\delta$ linearly independent vectors $\mathbf{y}=\mathbf{w} \otimes \overline{\mathbf{w}}$ in the kernel of $\hat{P}$. Hence, it is necessary that $\operatorname{dim} \operatorname{ker} \hat{P} \geq \delta$. Since $\hat{P}:(n-1) \times d^{2}$, we also have $\operatorname{dim} \operatorname{ker} \hat{P} \geq d^{2}-(n-1)$. To be able to detect $\delta$ from $\operatorname{dim} \operatorname{ker} \hat{P}$, we have to require that, at least, $n \geq d^{2}+1$.

Proposition 5: Let $\delta$ be the number of CM signals in $X$, and suppose $n>d^{2}$. Then $\operatorname{dim} \operatorname{ker} \hat{P} \geq \delta$. Generically, dimker $\hat{P}=\delta$.

Proof: See Appendix B.

In the proof it is shown that the occurrence of the non-generic case where $\hat{\delta}>\delta$ is independent of the propagation environment and can only happen if there are specific phase relations between the signals. Explicit examples can be constructed for the case of two CM signals $\mathbf{s}_{1}, \mathbf{s}_{2}$ : a rank deficiency occurs if and only if there are constants $\alpha, c \in \mathbb{C}$ such that for each sample point $k$,

$$
\left(\mathbf{s}_{1}\right)_{k}\left(\overline{\mathbf{s}}_{2}\right)_{k}+\alpha\left(\mathbf{s}_{2}\right)_{k}\left(\overline{\mathbf{s}}_{1}\right)_{k}=c .
$$

Writing $\left(\mathbf{s}_{2}\right)_{k}=\left(\mathbf{s}_{1}\right)_{k} \exp j \phi_{k}$, where $\phi_{k}$ is the phase difference between the two signals, this reduces to

$$
e^{-j \phi_{k}}+\alpha e^{j \phi_{k}}=c .
$$

For every choice of constants $\alpha, c$, there are at most two values for $\phi_{k}$ such that the equation holds. Hence, a degeneracy can occur only for BPSK-type (two state) signals sampled at the symbol rate. E.g. for BPSK, $\phi_{k} \in\{0, \pi\}$, so that there are constants $(\alpha=-1, c=0)$ for which (15) holds for all $k$. A problem also occurs for MSK signals, which are \pm 1 for even $k$ and $\pm j$ for odd $k$. These degeneracies go away when the signals are fractionally sampled, because $\phi_{k}$ then also assumes intermediate values.

\section{B. Computation of $W$}

We will assume from now on that $\operatorname{dim} \operatorname{ker} \hat{P}=\delta$ is equal to the number of CM signals in $X$. The CM problem is solved once we have found all $\delta$ independent parameter vectors $\left[\alpha_{1}, \cdots, \alpha_{\delta}\right]$ that make the generalized matrix pencil (14) rank one and hermitian. This problem is in essence a generalized eigenvalue problem. Indeed, if $d=\delta=2$, then there are two matrices $Y_{1}$ and $Y_{2}$, each of size $2 \times 2$, and we have to find $\lambda=\alpha_{2} / \alpha_{1}$ such that $Y_{1}+\lambda Y_{2}$ has its rank reduced by one (to become one). For larger $\delta$, there are more than two matrices, and the rank should be reduced to one by taking linear combinations of all of them. This can be viewed as an extension of the generalized eigenvalue problem.

From the opposite perspective, suppose that the solutions of the CM problem are $\mathbf{w}_{1}, \cdots, \mathbf{w}_{\delta}$. We already showed that $\mathbf{w}_{1} \otimes$ $\overline{\mathbf{w}}_{1}, \cdots, \mathbf{w}_{\delta} \otimes \overline{\mathbf{w}}_{\delta}$ is a linearly independent set of vectors; together they are a basis of the kernel of $\hat{P}$. Moving to matrices, each of the matrices $Y_{1}, \cdots, Y_{\delta}$ is a different (independent) linear combination of the "pencil basis" $\mathbf{w}_{1}^{*} \mathbf{w}_{1}, \cdots, \mathbf{w}_{\delta}^{*} \mathbf{w}_{\delta}$, i.e.,

$$
\begin{gathered}
Y_{1}=\lambda_{11} \mathbf{w}_{1}^{*} \mathbf{w}_{1}+\lambda_{12} \mathbf{w}_{2}^{*} \mathbf{w}_{2}+\cdots+\lambda_{1 \delta} \mathbf{w}_{\delta}^{*} \mathbf{w}_{\delta}=W^{*} \Lambda_{1} W \\
Y_{2}=\lambda_{21} \mathbf{w}_{1}^{*} \mathbf{w}_{1}+\lambda_{22} \mathbf{w}_{2}^{*} \mathbf{w}_{2}+\cdots+\lambda_{2 \delta} \mathbf{w}_{\delta}^{*} \mathbf{w}_{\delta}=W^{*} \Lambda_{2} W \\
\vdots \\
Y_{\delta} \stackrel{\vdots}{=} \lambda_{\delta 1} \mathbf{w}_{1}^{*} \mathbf{w}_{1}+\lambda_{\delta 2} \mathbf{w}_{2}^{*} \mathbf{w}_{2}+\cdots+\lambda_{\delta \delta} \mathbf{w}_{\delta}^{*} \mathbf{w}_{\delta} \stackrel{\doteq}{=} W^{*} \Lambda_{\delta} W
\end{gathered}
$$

where

$$
W=\left[\begin{array}{c}
\mathbf{w}_{1} \\
\vdots \\
\mathbf{w}_{\delta}
\end{array}\right], \quad \Lambda_{k}=\left[\begin{array}{ccc}
\lambda_{k 1} & & \mathbf{0} \\
& \ddots & \\
\mathbf{0} & & \lambda_{k \delta}
\end{array}\right] \quad(k=1, \cdots, \delta) .
$$

Hence, by the existence of a solution to the CM problem, there must be a matrix $W$ whose inverse simultaneously diagonalizes $Y_{1}, \cdots, Y_{\delta}$. (Its uniqueness is, in fact, proven in [15] for the case of hermitian matrices $Y_{k}$.) This makes problem $\mathrm{P} 2$ equivalent to the following problem.

Theorem 6: Suppose $n>d^{2}$ and $\operatorname{dim} \operatorname{ker}(\hat{P})=\delta$. Then the $\mathrm{CM}$ factorization problem $\mathrm{P} 1$, or $\mathrm{P} 2$, is equivalent to a simultaneous diagonalization problem: find $W: \delta \times d($ full rank $\delta$ ) such that

$$
\begin{aligned}
Y_{1} & =W^{*} \Lambda_{1} W \\
Y_{2} & =W^{*} \Lambda_{2} W \\
& \vdots \\
Y_{\delta} & \stackrel{ }{=} W^{*} \Lambda_{\delta} W
\end{aligned} \quad\left(\Lambda_{1}, \cdots, \Lambda_{\delta} \in \mathbb{C}^{d \times d}, \text { diagonal }^{3}\right)
$$

In general, $Y_{1}$ and $Y_{2}$ are $d \times d$ matrices of rank $\delta$, and not less than $\delta$. In this case, a generalized eigenvalue decomposition of just $Y_{1}$ and $Y_{2}$ will already determine $W$ : there exist matrices $M, N$ (invertible) such that

$$
\begin{aligned}
& M^{*} Y_{1} N=\Lambda_{1} \\
& M^{*} Y_{2} N=\Lambda_{2}
\end{aligned}
$$

where $\Lambda_{1}, \Lambda_{2}$ are diagonal matrices, size $d \times d$, each with $\delta$ nonzero entries. Reducing these matrices to $\delta \times \delta$ diagonal matrices with non-zero entries on the diagonal, and trimming $M$ and $N$ likewise to full rank $\delta \times d$ matrices, we obtain the decomposition

$$
\begin{aligned}
& \hat{M}^{*} Y_{1} \hat{N}=\hat{\Lambda}_{1} \\
& \hat{M}^{*} Y_{2} \hat{N}=\hat{\Lambda}_{2} .
\end{aligned}
$$

$\hat{M}$ and $\hat{N}$ are unique up to equal permutations of their columns and (possibly different) right diagonal invertible factors. This uniqueness implies that, after a suitable diagonal scaling, we can arrange it such that $\hat{M}=\hat{N}=W^{\dagger}$, or $W=\hat{N}^{\dagger}$, with each row of $W$ having norm $n^{1 / 2}$.

For the case where $Y_{1}$ and $Y_{2}$ are not of rank $\delta$, it is possible that they do not fully determine $W$, so that the other $Y_{k}$ also have to be taken into account. It is obvious that it is possible to obtain $W$ also in this case, but we omit the details of this more general procedure at this point. Numerically, it is better to take all $Y_{k}$ into account in all cases, and this is of course preferable in the presence of noise as well. Such an algorithm is described in the next section.

Hence, we have shown at this point that in absence of noise, the $\mathrm{CM}$ problem is in fact a generalized eigenvalue problem and can be solved explicitly.

\footnotetext{
${ }^{3}$ If we make sure that the $Y_{k}$ are hermitian by our choice of the vec-operation, then the $\Lambda_{k}$ are real.
} 


\section{THE CM PROBLEM WITH ADDITIVE NOISE}

\section{A. Equivalent optimization problem}

With noise added to the data,

$$
X=A S+N,
$$

an exact decomposition of $X$ as $X=A S$ is no longer possible. To recover the CM signals, we first define a (squared) distance function of signals to the set of CM signals,

$$
\operatorname{dist}(\mathbf{s}, \mathcal{C M})=\sum_{k=1}^{n}\left(\left|(\mathbf{s})_{k}\right|^{2}-1\right)^{2} .
$$

In terms of this distance, the problem can be posed as finding $\delta$ independent signals $\mathbf{s}$ that are minimizers of

$$
\min \left\{\operatorname{dist}(\mathbf{s}, \mathcal{C M}) \mid \mathbf{s} \in \operatorname{row}^{\prime}(X)\right\},
$$

where $\operatorname{row}^{\prime}(X)$ is the estimated row span of $S$, which we will take to be the principal row span of $X$ as determined using an SVD. Thus let $X=U \Sigma V$ as before, and let $d$ be the number of singular values of $X$ that are significantly larger than zero. The detection of $d$ from the singular values is relatively straightforward if the noise power and the statistical distribution of the noise is known, but notoriously non-trivial otherwise (cf. [42] and references therein; we do not go into details here). The rows of $V$ corresponding to these singular values form an orthogonal basis of the principal row span of $X$, and are collected in the matrix $\hat{V}$. The matrix $P$ and $\hat{P}$ can be constructed from $\hat{V}$ as in section II-C. The following proposition is a result of expressing the cost function in equation (18) in terms of $\hat{P}$ and $\mathbf{w}$.

Proposition 7: The CM problem with noise (equation (18)) is solved by finding the set of all linearly independent minimizers $\mathbf{y}$ of

$$
\varepsilon_{\mathbf{y}}^{2}:=\min _{\mathbf{y}}\|\hat{P} \mathbf{y}\|^{2},
$$

subject to $\mathbf{y}=\mathbf{w} \otimes \overline{\mathbf{w}},\|\mathbf{w}\|=n^{1 / 2}$. For each such $\mathbf{y}$, the corresponding signal $\mathbf{s}$ is $\mathbf{s}=(c \mathbf{w}) \hat{V}$, where the corrective scaling $c$ is given by $c^{2}=n /\left(n+\varepsilon_{\mathbf{y}}^{2}\right)$.

Proof: See Appendix B.

The correction of $\mathbf{w}$ by $c$ is close to 1 and of no importance in practice, as it will only scale the amplitude of the corresponding signal s.

Minimizing (19) with the given conditions on $\mathbf{y}$ undoubtedly requires some iterative method, but the route set out by the solution of the noiseless case will provide accurate initial points for such a method. Thus, we first compute a basis of orthogonal vectors $\mathbf{y}_{k}$ that solve (19) without structural constraint: as before, these follow from an SVD of $\hat{P}$ as the right singular vectors corresponding to the smallest $\hat{\delta}$ singular values (the numerical kernel of $\hat{P}$ ). The number of CM signals is estimated from the number of singular values that are "significantly smaller" than the others; a suitable threshold level is given in (30) later in this paper. The next step is to unstack the vectors $\mathbf{y}_{k}$ into corresponding matrices $Y_{k}=\operatorname{vec}^{-1}\left(\mathbf{y}_{k}\right)$, and subsequently impose the required Kronecker structure onto these matrices: linear combinations of the $Y_{k}$ should result in matrices that are close to rank-1 hermitian matrices of the form $\mathbf{w}^{*} \mathbf{w}$, i.e.,

$$
\alpha_{1} Y_{1}+\cdots+\alpha_{\delta} Y_{\delta}=Y \simeq \mathbf{w}^{*} \mathbf{w} .
$$

Again, it is not clear how to solve such a problem exactly. In the noise-free case, the solution was given by a simultaneous diagonalization of $\delta$ matrices $Y_{1}, \cdots, Y_{\delta}$, but it could be found from only two of them. We now extend the simultaneous diagonalization approach to the noisy case, this time taking all available $Y_{k}$ into account.

\section{B. Simultaneous diagonalization as a super-generalized Schur problem}

Assume, for the moment, that there is no noise added to $X$. As we have seen in theorem 6 , the matrix $W \in \mathbb{C}^{\delta \times d}$ that we try to find is full rank and such that

$$
\begin{aligned}
& Y_{1}=W^{*} \Lambda_{1} W \\
& Y_{2}=W^{*} \Lambda_{2} W \\
& \quad \vdots \\
& Y_{\delta} \stackrel{ }{=} W^{*} \Lambda_{\delta} W
\end{aligned} \quad\left(\Lambda_{1}, \cdots, \Lambda_{\delta} \in \mathbb{C}^{\delta \times \delta}, \text { diagonal }\right)
$$

With noise, we can try to find $M=W^{\dagger}$ to simultaneously make $M^{*} Y_{1} M, \cdots, M^{*} Y_{\delta} M$ as much diagonal as possible. Because $M$ is not unitary, the fact that it has to have full rank is hard to quantify, and it makes sense to rewrite this $\delta$-generalized eigenvalue problem as a $\delta$-generalized Schur decomposition. We first explain the procedure for the noise-free case. Bring in a $\mathrm{QR}$ factorization of $W^{*}$ and an RQ decomposition of $W$ :

$$
W^{*}=Q^{*} R^{\prime}, \quad W=R^{\prime \prime} Z^{*}
$$

where $Q, Z$ are unitary $d \times d$ matrices, and $R^{\prime} \in \mathbb{C}^{d \times \delta}, R^{\prime \prime} \in \mathbb{C}^{\delta \times d}$ are upper triangular. The factorizations are of course related, but we will ignore this for the moment. If $\delta<d$, then we can arrange that only the leading $\delta \times \delta$ blocks of $R^{\prime}$ and $R^{\prime \prime}$ are non-zero (and non-singular). Substitution into (21) leads to

$$
\begin{aligned}
& Q Y_{1} Z=R_{1} \quad\left(R_{1}, \cdots, R_{\delta} \in \mathbb{C}^{d \times d}, \text { upper triang. }\right) \\
& Q Y_{2} Z=R_{2} \\
& Q Y_{\delta} Z \stackrel{\vdots}{=} R_{\delta}
\end{aligned}
$$

with

$$
\begin{aligned}
R_{1} & =R^{\prime} \Lambda_{1} R^{\prime \prime} \\
R_{2} & =R^{\prime} \Lambda_{2} R^{\prime \prime} \\
& \vdots \\
R_{\delta} & =R^{\prime} \Lambda_{\delta} R^{\prime \prime} .
\end{aligned}
$$

Only the top-left $\delta \times \delta$ block of each $R_{k}$ is non-zero. In addition, each of these blocks is non-singular. Hence, there exists $Q, Z$ such that $Q Y_{k} Z$ is upper triangular, for $k=1, \cdots, \delta$, which is a generalized Schur decomposition, but for $\delta$ matrices rather than two. With this decomposition, it is seen that a parameter vector $\left[\alpha_{1} \cdots \alpha_{\delta}\right]$ satisfies (20) only if

$$
\alpha_{1} R_{1}+\cdots+\alpha_{\delta} R_{\delta} \text { is rank } 1 .
$$

With the model of $R_{1}, \cdots, R_{\delta}$ in (23), we obtain that, equivalently,

$$
\alpha_{1} \Lambda_{1}+\cdots+\alpha_{\delta} \Lambda_{\delta} \text { is rank } 1
$$

Since all the $\Lambda_{k}$ are diagonal, the $\alpha_{k}$ are straightforward to compute: only one entry of the diagonal matrix $\alpha_{1} \Lambda_{1}+\cdots+\alpha_{\delta} \Lambda_{\delta}$ 
can be non-zero. Setting this entry equal to one, all possible parameter vectors $\left[\alpha_{1} \cdots \alpha_{\delta}\right]$ follow by constructing a matrix whose columns consist of the diagonal entries of the $\Lambda_{k}$,

$$
\Lambda=\left[\begin{array}{ccc}
\left(\Lambda_{1}\right)_{11} & \cdots & \left(\Lambda_{1}\right)_{\delta \delta} \\
\vdots & & \vdots \\
\left(\Lambda_{\delta}\right)_{11} & \cdots & \left(\Lambda_{\delta}\right)_{\delta \delta}
\end{array}\right]
$$

The rows of $\Lambda^{-1}$ are the independent vectors $\left[\begin{array}{lll}\alpha_{1} & \cdots & \alpha_{\delta}\end{array}\right]$. A straightforward generalization shows that we do not need to compute the factorization (23), as the parameters can be computed directly from the main diagonals of $R_{k}$ without knowing the $\Lambda_{k}$ :

Proposition 8: For given $Y_{1}, \cdots, Y_{\delta}$, assume the decomposition (22) and the existence of decomposition (23). All independent parameter vectors $\left[\alpha_{1} \cdots \alpha_{\delta}\right]$ such that $Y:=\alpha_{1} Y_{1}+\cdots+$ $\alpha_{\delta} Y_{\delta}$ has rank 1 are given by the rows of $A$ :

$$
A=R^{-1}, \quad R=\left[\begin{array}{ccc}
\left(R_{1}\right)_{11} & \cdots & \left(R_{1}\right)_{\delta \delta} \\
\vdots & & \vdots \\
\left(R_{\delta}\right)_{11} & \cdots & \left(R_{\delta}\right)_{\delta \delta}
\end{array}\right] .
$$

Proof: Because the $R_{k}$ are upper triangular, a necessary condition for (24) to hold is that the resulting matrix has a main diagonal with at most one non-zero entry. But, in view of the existence of factorization (23) with $R^{\prime}, R^{\prime \prime}$ having non-singular main diagonals, it cannot happen that the main diagonal of the result is all zero.

Note that proposition 8 by itself does not ensure that $Y$ is hermitian, unless e.g., all $Y_{i}$ 's are hermitian and all $R_{i}$ 's are real. This feature is a side effect: the set of $\delta$ independent solutions $Y$ is in our case unique, and it suffices to enforce the rank-1 property.

Factoring each of the $\delta$ rank-1 matrices that is obtained in this way gives $\delta$ independent vectors $\mathbf{w}$, which form the rows of the matrix $W$ that we were looking for in equation (21). Hence, in the noise-free case, the computation of a "super-generalized" Schur decomposition, i.e., two unitary matrices $Q, Z$, gives the solution to the simultaneous diagonalization problem. Although it seems at first sight that we have doubled the number of parameters to estimate (two matrices $Q, Z$, rather than one matrix $W$ ), this is not true: the fact that the matrices are unitary makes that the total number of parameters to estimate is precisely the same. However, the constraint that $Q, Z$ be unitary is a desirable condition, whereas the fact that $W$ must have full rank is difficult to handle.

We now return to the case where the data matrix $X$ is distorted by noise. In this case, there is no unitary $Q, Z$ which simultaneously makes all matrices $Y_{k}$ exactly upper triangular. However, we can try to find $Q, Z$ to make these matrices as much upper triangular as possible, by minimizing the Frobenius norm of the residual lower triangular entries. One approach for doing this is described in section IV-C below. It is an extension to more than two matrices of the usual QZ iteration for computing the generalized Schur decomposition of two matrices. There are several other approaches for solving simultaneous diagonalization problems as well, as discussed in that subsection.

With $Q, Z$ and hence $R_{1}, \cdots, R_{\delta}$ obtained this way, we can compute all independent parameter vectors $\left[\alpha_{1} \cdots \alpha_{\delta}\right]$ as in proposition 8 . Each parameter vector gives a matrix $Y$, approximately of the form $Y \simeq \mathbf{w}^{*} \mathbf{w}$, and each $\mathbf{w}$ can be estimated as the singular vector corresponding to the largest singular value of each $Y$. It remains to scale $\mathbf{w}$ to ensure that $\|\mathbf{w}\|=n^{1 / 2}$.

The above scheme provides an approximate solution to the problem in theorem 7, i.e., the CM factorization problem with additive noise. The algorithm is summarized in figure 2; we call it the ACMA. It is not clear in what sense the solution approximates the optimal solution; however, it finds the exact solution if there is no noise, and simulations give very accurate results for moderate noise levels or large $n$. For high noise levels, closely spaced signals, or small $n$, the vectors $\mathbf{w}$ that are obtained by the above procedure can be used as initial starting points in a Gerchberg-Saxton iteration, which effectively searches for the minima of (18). Since these starting points are accurate, a few iterations suffice, and independent signals are almost always obtained, except in severely ill-conditioned cases. Examples of the application of the algorithm to simulated and measured data are given in section $\mathrm{V}$.

\section{Super-generalized Schur decomposition}

In this subsection, we describe a possible approach to the super-generalized Schur decomposition problem: for given matrices $Y_{1}, \cdots, Y_{\delta}$, find $Q, Z$ (unitary) such that

$$
\begin{aligned}
Q Y_{1} Z & =R_{1} \\
& \vdots \\
Q Y_{\delta} Z & =R_{\delta}
\end{aligned}
$$

where $R_{1}, \cdots, R_{\delta}$ are as much upper triangular as possible. Our approach is to modify the standard QZ iteration method used for computing the Schur decomposition of two matrices so that it works for more than two matrices. There are several ways to do this. We will present a variant that treats all matrices $Y_{1}, \cdots Y_{\delta}$ equally.

The QZ iteration for computing the Schur decomposition of two matrices [40] starts with setting $Q^{(0)}=I, Z^{(0)}=I$. At the $k$-th iteration step, a unitary matrix $Q^{(k)}$ is computed such that $Q^{(k)}\left(Y_{1} Z^{(k-1)}\right)$ is upper triangular, and a unitary matrix $Z^{(k)}$ is computed to make $\left(Q^{(k)} Y_{2}\right) Z^{(k)}$ upper triangular. As an extension to more than two matrices, we propose the following two step iteration. Denote by $\|\cdot\|_{L F}$ the Frobenius norm of the strictly lower triangular part of a matrix.

$$
\begin{aligned}
& \text { [Extended QZ iteration] } \\
& Q^{(0)}=I, Z^{(0)}=I \\
& \text { for } k=1,2, \cdots \\
& \quad \text { a. Find } Q^{(k)} \text { (unitary) to minimize } \\
& \quad\left\|Q^{(k)}\left(Y_{1} Z^{(k-1)}\right)\right\|_{L F}^{2}+\cdots+\left\|Q^{(k)}\left(Y_{\delta} Z^{(k-1)}\right)\right\|_{L F}^{2}, \\
& \quad \text { b. find } Z^{(k)} \text { (unitary) to minimize } \\
& \left.\quad\left\|\left(Q^{(k)} Y_{1}\right) Z^{(k)}\right\|_{L F}^{2}+\cdots+\|\left(Q^{(k)} Y_{\delta}\right) Z^{(k)}\right) \|_{L F}^{2} .
\end{aligned}
$$

Each of the two steps in the iteration poses a least squares problem with an exact solution, which might however be hard to find. The customary idea in such situations is to find an approximate solution to each of the steps, and rely on the outer iteration to provide convergence.

\footnotetext{
${ }^{4}$ A different suitable initialization follows from a Schur decomposition of just $Y_{1}$ and $Y_{2}$.
} 
Given a matrix $X=A S+N \in \mathbb{C}^{m \times n}$. An estimate of $S \in \mathcal{C M}$ is obtained as follows:

1. Estimate $\operatorname{row}(X)$ :

a. Compute $\operatorname{SVD}(X): X=: U \Sigma V$

b. Estimate $d=\operatorname{rank}(X)$ from $\Sigma$ : the number of signals

c. $\hat{V}=$ first $d$ rows of $V$

2. Estimate $\operatorname{ker}(\hat{P})$, which summarizes all $\mathrm{CM}$ conditions:

a. Construct $\hat{P}:(n-1) \times d^{2}$ from $\hat{V}$ :

$$
\begin{aligned}
& \hat{V}=:\left[\mathbf{v}_{1} \cdots \mathbf{v}_{n}\right] \\
& P:=\left[\operatorname{vec}\left(\mathbf{v}_{1} \mathbf{v}_{1}^{*}\right) \cdots \operatorname{vec}\left(\mathbf{v}_{n} \mathbf{v}_{n}^{*}\right)\right]^{T} \\
& {\left[\begin{array}{c}
\hat{\mathbf{p}}_{1} \\
\hat{P}
\end{array}\right]:=Q P, \text { with } Q \text { as in }(11)}
\end{aligned}
$$

b. Compute $\operatorname{SVD}(\hat{P}): \hat{P}=: U_{p} \Sigma_{p} V_{p}^{*}$

c. Estimate $\delta=\operatorname{dim} \operatorname{ker}(\hat{P})$ from $\Sigma_{p}$ : the number of CM signals

d. $\left[\mathbf{y}_{1} \cdots \mathbf{y}_{\delta}\right]:=$ last $\delta$ columns of $V_{p}$

3. Solve the simultaneous diagonalization problem (21):

a. $Y_{1}=\operatorname{vec}^{-1}\left(\mathbf{y}_{1}\right), \cdots, Y_{\delta}=\operatorname{vec}^{-1}\left(\mathbf{y}_{\delta}\right)$

b. Find $Q, Z$ to make $R_{1}:=Q Y_{1} Z, \cdots, R_{\delta}:=Q Y_{\delta} Z$ approximately upper (section IV-C)

c. From $R_{1}, \cdots, R_{\delta}$, compute all vectors $\left[\alpha_{k 1} \cdots \alpha_{k \delta}\right], k=1, \cdots, \delta$

s.t. $\hat{Y}_{k}:=\alpha_{k 1} Y_{1}+\cdots+\alpha_{k \delta} Y_{\delta}$ is approximately rank 1 (proposition 8)

4. Recover the signals: for each $\hat{Y}_{k}$ :

a. Compute $\mathbf{w}_{k}$ such that $\hat{Y}_{k} \simeq: \mathbf{w}_{k}^{*} \mathbf{w}_{k}$

b. scale $\mathbf{w}_{k}$ such that $\left\|\mathbf{w}_{k}\right\|=n^{1 / 2}$

c. $\mathbf{s}_{k}:=\mathbf{w}_{k} \hat{V}$

(d. perform a few Gerchberg-Saxton iterations, as in equation (3))

The vectors $\mathbf{s}_{1}, \cdots, \mathbf{s}_{\delta}$ are the rows of $S$.

Fig. 2. The ACMA (analytic CM factorization algorithm). The vectoring operations in steps $2 \mathrm{a}$ and $3 \mathrm{a}$ may be replaced by hermitian vectoring operations (see Appendix A).

To describe the approximate solution to step a (or b, which is similar), suppose that, at the $k$-th stage, we have matrices $R_{1}:=$ $Q^{(k-1)} Y_{1} Z^{(k-1)}, \cdots, R_{\delta}:=Q^{(k-1)} Y_{\delta} Z^{(k-1)}$, not yet upper triangular, and we have to find a unitary matrix $Q$ that minimizes the belowdiagonal norm of $Q R_{1}, \cdots, Q R_{\delta}$. Recall that for a single matrix $R_{1}$, a QR factorization gives the solution, and is obtained as a product of Householder rotations $H_{1}, \cdots, H_{d-1}$, where a single $H_{i}$ maps the below-diagonal entries of the $i$-th column of the matrix to zero. This approach may be mimicked for the simultaneous triangularization of a set of matrices, although we can only try to make the below-diagonal entries small. Thus, $Q$ in step a is obtained as the product of $d-1$ more elementary unitary matrices

$$
Q=\left[\begin{array}{c|c}
I_{d-2} & 0 \\
\hline 0 & H_{d-1}
\end{array}\right] \cdots\left[\begin{array}{c|c}
1 & 0 \\
\hline 0 & H_{2}
\end{array}\right] H_{1} .
$$

The first factor, $H_{1}$, is designed to simultaneously minimize the below-diagonal norms of only the first column of each of the matrices $R_{1}, \ldots, R_{\delta}$. Similarly, $H_{k}$ is used to minimize the belowdiagonal norm of all the $k$-th columns. Denote by $\left(R_{1}\right)_{1}$ the first column of $R_{1}$, and similarly for the other $R_{k}$ 's, then

$$
H_{1}\left[\left(R_{1}\right)_{1} \cdots\left(R_{\delta}\right)_{1}\right]=\left[\begin{array}{ccc}
* & \cdots & * \\
\hline E
\end{array}\right]
$$

where $[* \ldots *]$ is the first row of the result, and $E$ contains the remaining rows. The objective is to find $H_{1}$ such that $\|E\|_{F}$ is minimized. The solution is not unique, but a possible $H_{1}$ follows di- rectly from an SVD:

$$
\left[\left(R_{1}\right)_{1} \cdots\left(R_{\delta}\right)_{1}\right]=: U \Sigma V^{*} \Rightarrow H_{1}=U^{*} .
$$

Indeed, for this choice of $H_{1}$, we have $\|E\|_{F}^{2}=\sigma_{2}^{2}+\cdots \sigma_{\delta}^{2}$, which is as small as we can hope for.

After $H_{1}$ has been computed and applied to $R_{1}, \cdots, R_{\delta}$, we have obtained new matrices $R_{1}^{\prime}, \cdots, R_{\delta}^{\prime}$, with the below-diagonal norm of the first columns minimized. The next factor, $\mathrm{H}_{2}$, is used to minimize the below-diagonal norm of the second columns of these matrices. As $\mathrm{H}_{2}$ is unitary and does not affect the first rows of $R_{1}^{\prime}, \cdots, R_{\delta}^{\prime}$, this will not change the below-diagonal norm of the first columns. In fact, $H_{2}$ can be found in precisely the same way as $H_{1}$ by looking at the reduced problem where we act on $R_{1}^{\prime}, \cdots, R_{\delta}^{\prime}$ with their first rows and columns removed. The matrices $H_{3}, \cdots, H_{d-1}$ follow in turn.

The reason that this does not necessarily provide the optimal solution to the LS problem of step a is that $H_{1}$ only looks at the first columns of the matrices $R_{k}$, and might introduce potentially large entries in the below-diagonal part of subsequent columns. It is not even guaranteed that the below-diagonal norm is lower than before. Note that this is nothing new: the same happens in the original QZ iteration for two matrices, and nonetheless it converges (except perhaps for strongly nonnormal problems).

The resulting QZ iteration (26) is observed in simulations to converge fast, usually quadratically in $3-5$ iterations. At this point, there is no proof of convergence. Hints for a possible proof might be provided by convergence proofs for the standard QZ iteration. Because the inner loop consist of SVDs, the scheme is 
only practical if $d$ is small, which is certainly the case for the currently envisioned applications.

\section{Remark}

While this paper was in review, we learned about other approaches to the super-generalized Schur decomposition, and to simultaneous diagonalization problems in general. These problems are not entirely new to the SP community: in the context of blind beamforming of non-Gaussian signals, analysis of the fourth-order cumulants of the data matrix has led to problems of the form (21), viz. [18,43], as well as related problems of the form $Q^{*} X_{i} Q=\Lambda_{i}, i=1, \cdots, d$ with the $X_{i}$ hermitian, $Q$ unitary and all $\Lambda_{i}$ close to diagonal, viz. [17]. The fact that $Q$ is unitary instead of just non-singular is a consequence of the statistical expectation operator: with infinite data, our $W$ would be unitary as well (cf. footnote 2). In [18,43], a whitening transformation derived from the data covariance matrix immediately reduces the problem to $Q^{*} X_{i} Q=\Lambda_{i}$ as well.

Overviews of several such problems are given in [44,45]. The algorithms in [44] are of Jacobi-type, and intended for solving $Q^{*} X_{i} Q=\Lambda_{i}$ and some structured variants. A similar Jacobi algorithm is proposed in [17]. Such Jacobi iterations are readily set up for the generalized Schur decomposition (25) as well, although one has to be careful about "outer" and "inner" rotations to ensure convergence; cf. [46]. The (real-valued) QZ problem is considered in [45], and solved using isospectral flows, which results in a steepest gradient-type algorithm. For the diagonalization problem (21) with positive definite matrices $Y_{i}$, the "nonorthogonal $\mathrm{FG}^{+}$" algorithm of [47] may be used. The orthogonal variant of this algorithm is a generalization of the cyclic Jacobi algorithm. Note that in our application, the $Y_{i}$, even if they are constructed to be hermitian, are not necessarily positive, although we may try to find linear combinations that are positive. The approach in $[18,43]$ is to find one such positive combination, then try to glean $W$ from a Cholesky decomposition (or Schur decomposition, after a whitening transformation) of this single matrix. Numerically, this is likely to be suboptimal, because in the end only two matrices determine the decomposition. So far, none of the above approaches has proven convergence, but reported experimental results are invariably positive.

\section{Computational complexity}

We briefly investigate the computational complexity of the proposed algorithm (figure 2). The ACMA consists of mainly three computational steps: an SVD of $X$ (size $m \times n)$, an SVD of $\hat{P}\left(\right.$ size $\left.n-1 \times d^{2}\right)$, and a simultaneous diagonalization of $\delta$ matrices $Y_{i}$ of size $d \times d$. The second SVD is the most expensive and has order $n\left(d^{2}\right)^{2}$ operations. Since we require $n>m^{2}$, and $m \geq d$, the complexity of this step is at least $\mathcal{O}\left(d^{6}\right)$. In comparison, the first SVD has $\mathcal{O}\left(m^{2} n\right) \geq \mathcal{O}\left(d^{4}\right)$ operations, and the complexity of the simultaneous diagonalization step is also $\mathcal{O}\left(d^{4}\right)$. This implies that $d$ cannot be very large and that the algorithm is too complex for equalization purposes (where sometimes $d>100$ is taken). ${ }^{5}$ Since only subspaces are needed but not the individual

\footnotetext{
${ }^{5}$ As mentioned before, the CM equalization problem satisfies the same model, but has different properties: the data matrix has a Hankel structure, and, in principle, it suffices to find only one solution. In this case, a combination with other (intersection-type) algorithms that make use of this structure is probably
}

singular vectors, the SVDs may be replaced by any other principal subspace estimator, such as provided by the Schur subspace estimation (SSE) method [48], the URV updating [49], the PAST method [50], the FSD [51], or the FST [52]. The latter three algorithms can also exploit the fact that only $d$ kernel vectors out of $d^{2}$ singular vectors are needed, which gives rise to significant savings. In addition, all above-mentioned methods allow for efficient updating of the subspaces for increasing $n$, so that the ACMA algorithm may be transformed from a block-algorithm into an adaptive algorithm. (Interesting complications arise because the two SVDs operate in conjunction. A more detailed analysis of the possibilities is beyond the scope of the present paper.)

To illustrate the computational requirements in a more quantitative manner, we consider a replacement of the SVD by the SSE. Without updating, one implementation of the SSE (the "SSE-2" [53]) has a complexity of about $m^{2} n$ complex multiplications and $2 m^{2} n$ complex rotations (for a matrix of size $n \times m$ ). Assuming no special purpose rotation processors, we set 1 complex rotation equal to 4 complex multiplications, and 1 complex multiplication equal to 4 real floating point operations (flop). In that case the SSE of $X$ takes $36 \mathrm{~m}^{2} n$ real flop, and (since $\hat{P}$ can be transformed to a real matrix) the SSE of $\hat{P}$ takes $9\left(d^{2}\right)^{2} n$ real flop, so that the complexity of ACMA is

$$
\text { ACMA : } 9 d^{4} n+36 m^{2} n \text { real flop. }
$$

In comparison, the complexity of the Gerchberg-Saxton algorithm (GSA) (3) is mainly determined by a loop containing two complex matrix multiplications, $W \cdot X$ and $S \cdot X^{\dagger}$ (where $W: d \times m$, $X: m \times n, S: d \times n)$, not taking additional soft orthogonalization steps or restarts into account. Each of the multiplications has a complexity of $d m n$ complex operations. About 10 iterations of the inner loop are usually sufficient, although occasionally many more are needed. In addition, the computation of $X^{\dagger}=X^{*}\left(X X^{*}\right)^{-1}$ calls for about $2 m^{2} n$ complex multiplications (ignoring the inversion), or $8 m^{2} n$ real flop. Altogether, the complexity of GSA is approximately

GSA (CMA) : $4 \cdot 10 \cdot 2 d m n+8 m^{2} n=80 d m n+8 m^{2} n$ real flop.

The standard CMA may be viewed as an updating version of the GSA, where instead of iterating on the same data, new data is continuously introduced. It is not likely to converge faster than GSA, viz. [37], so that it has at least the complexity of (28). Table I gives a listing of (27) and (28) for a range of values of $m$ and $d$, in kflop per snapshot (i.e., $n=1$ ). It is seen that for up to 6 sources the complexity of the ACMA is comparable to the GSA.

\section{EXPERIMENTAL EVALUATION}

To assess the performance of the algorithm, we have applied it to a number of test matrices, based both on computer generated data and on real data collected from an experimental setup. The results are quite convincing. For example, the algorithm could find weight vectors to separate a superposition of four CM signals using 4 sensors and only 17 data samples, in

preferable. 
TABLE I

APPROXIMATE COMPUTATIONAL COMPLEXITY OF BLIND ALGORITHMS (KFLOP PER SNAPSHOT)

\begin{tabular}{|c|c|c|c|c|}
\hline \multicolumn{5}{|c|}{ ACMA (kflop $/ n$ ) } \\
\hline & $m=4$ & 6 & 8 & 10 \\
\hline$d=2$ & 0.7 & 1.4 & 2.4 & 3.7 \\
\hline 4 & 2.9 & 3.6 & 4.6 & 5.9 \\
\hline 6 & & 13.0 & 14.0 & 15.3 \\
\hline 8 & & & 39.2 & 40.5 \\
\hline 10 & & & & 93.6 \\
\hline
\end{tabular}

\begin{tabular}{|c|c|c|c|c|}
\hline \multicolumn{5}{|c|}{ GSA $(\mathrm{kflop} / n)$} \\
\hline & $m=4$ & 6 & 8 & 10 \\
\hline$d=2$ & 0.8 & 1.2 & 1.8 & 2.4 \\
\hline 4 & 1.4 & 2.2 & 3.1 & 4.0 \\
\hline 6 & & 3.2 & 4.4 & 5.6 \\
\hline 8 & & & 5.6 & 7.2 \\
\hline 10 & & & & 8.8 \\
\hline
\end{tabular}

well-conditioned cases even if each signal has a signal to background noise ratio of $5 \mathrm{~dB}$.

\section{A. Computer generated data}

We first study the performance of the algorithm on computer generated data. The set-up of this experiment is kept extremely simple on purpose. We simulate a uniform linear array of $m=4$ isotropic sensors, spaced $\lambda / 2$ apart, where $\lambda$ is the wavelength of the carrier frequency of the signals. ${ }^{6}$ The resulting main lobe has a beam width of approximately $26^{\circ}$. There are $d=4$ signals present, with angles of arrival $\theta_{1}=0^{\circ}$, varying $\theta_{2}\left(\theta_{2}=30^{\circ}\right.$, $\left.5^{\circ}\right), \theta_{3}=60^{\circ}, \theta_{4}=-20^{\circ}$. The number of $\mathrm{CM}$ signals among the four signals is varied from $\delta=4$ to $\delta=2$. The number of samples that are used is varied, too, and taken to be $n=100,26$, and 17. The CM signals that are generated are sequences of unitmodulus numbers with uniformly distributed random phase. The other (non-CM) signals are normally distributed random complex numbers, with zero mean and unit variance. The signals are scaled according to their relative SNRs.

In the first experiment, we consider the noiseless case. Figure $3(a)$ shows plots of the singular values of $\hat{P}$, for $\theta_{2}=30^{\circ}$, and with $d=\delta=4 \mathrm{CM}$ signals. In figure $3(b)$, only the first two signals are CM, the other two are Gaussian. In figure 3(c), the number of CM signals is again equal to 4 , but $\theta_{2}$ is taken to be $5^{\circ}$. It is seen that the number of zero singular values is precisely equal to the number of $\mathrm{CM}$ signals, as predicted by proposition 5. Changing the number of $\mathrm{CM}$ signals or moving the anglesof-arrival closer does not influence the distribution of the other singular values by much. In particular, the level of the smallest non-zero singular value stays roughly constant. The distribution of the non-zero singular values does change with $n$ : they tend to be located along slanted lines. For larger $n$, the graph flattens which facilitates detection. As there is no noise in the example so far, the CM signals can be retrieved without errors. To give an idea of the convergence speed of the extended QZ iteration, we list the total below-diagonal norm of the matrices $R_{k}$ in (25) after each iteration, for an instance of case $(a), n=17$ : $0.3,9 \cdot 10^{-6}, 4 \cdot 10^{-12}, 5 \cdot 10^{-18}$.

In the next series of experiments, the same set-up is used, but now we add normally distributed independent white noise to all samples. The SNR of each signal with respect to the noise level is set to $15 \mathrm{~dB}$ per antenna element. We first take all signals to be

\footnotetext{
${ }^{6}$ This information is not used in any way by the algorithm, and any other array geometry would have been suitable as well.
}

$\mathrm{CM}$, and $\theta_{2}=30^{\circ}$. The singular value plot of $\hat{P}$ is shown in figure $4(a)$. The previously zero singular values are now raised by some amount, but there still is a gap between the small and the larger ones. To evaluate how close the analytically computed weight vectors $\mathbf{w}_{1}, \cdots, \mathbf{w}_{4}$ are to the optimal solution of the problem (the minimizers of the distance function in (18)), these weight vectors are used as initial points in the Gerchberg-Saxton iteration (GSA), viz. equation (3). Figure 4(b) shows the computed average modulus error of $\mathbf{w} X$,

$$
\left[\frac{1}{n} \sum_{i=1}^{n}\left(\left|(\mathbf{w} X)_{i}\right|-1\right)^{2}\right]^{1 / 2}
$$

after each iteration step (solid line), for the case $n=17$. We have chosen this "1-2" norm rather than the "2-2" norm in (17) because it has a nicer physical interpretation (as the standard deviation of the modulus of signals), and because the convergence of the GSA is usually monotonic in this norm, but not in (17). It is seen that the post-processing hardly changes the computed $\mathbf{w}_{k}$, which is reflected by the horizontal lines: they are almost equal to the optimal values. For $n=26$ (not shown), the lines are perfectly straight. Although not clearly visible in $4(b)$, all four signals are resolved; because the signals have the same amplitude, the modulus error lines tend to overlap. The independence of the retrieved signals was checked by computing their covariance. The value of the modulus error is commensurate with the noise level and number of antennas: SNR $=15 \mathrm{~dB}$ translates to an expected modulus error of 0.063 in situations without co-channel interference.

Also shown in figure $4(b)$ is the performance of the Gerchberg-Saxton algorithm when started with random initial weight vectors (dashed lines), which would be the usual approach to the CM problem. It is seen that not always all signals are retrieved, that the convergence can be extremely slow, and that the algorithm sometimes converges to sub-optimal stationary values. We mention that for larger $n$ (say $n=100$ ), the local minima are usually not attractive, but recovering all independent signals remains an issue (solved to some extent by "soft-orthogonalization", as mentioned in the introduction, but this was not implemented in these simulations).

Essentially the same remarks can be made for the case where two out of four signals are CM signals (figure 5), and when the signal power of one or all of the signals is reduced to $5 \mathrm{~dB}$ (figures 6 and 7). The effect of a reduced signal power (or increased 

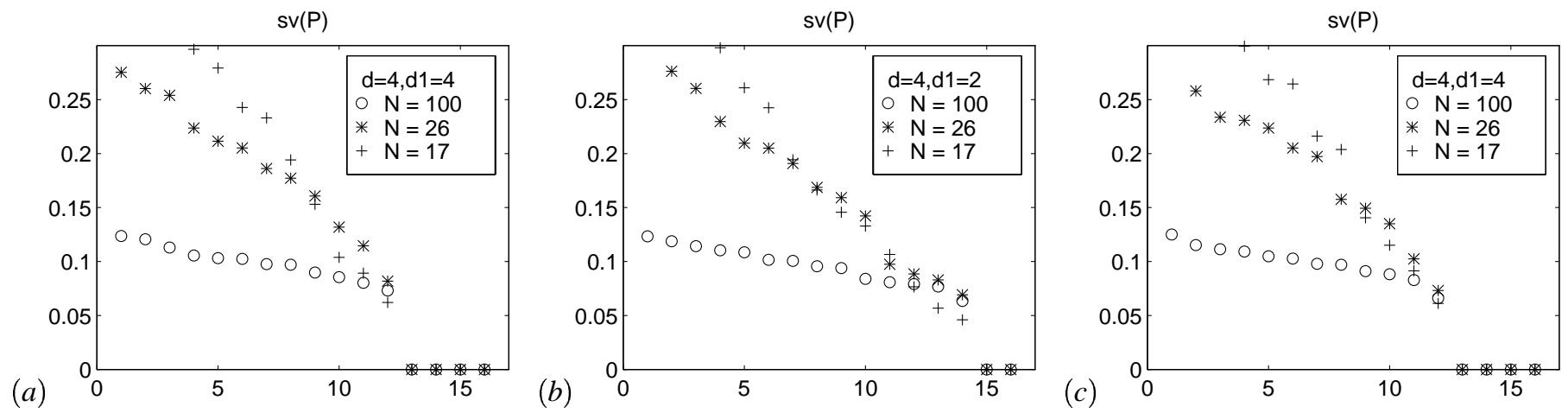

Fig. 3. Singular values of $\hat{P}$ : no noise, $\theta_{2}=30^{\circ}$, for $n=100,26,17$. (a) $4 \mathrm{CM}$ signals, $(b) 2 \mathrm{CM}$ signals. (c) Similar as $(a)$, but with $\theta_{2}=5^{\circ}$.

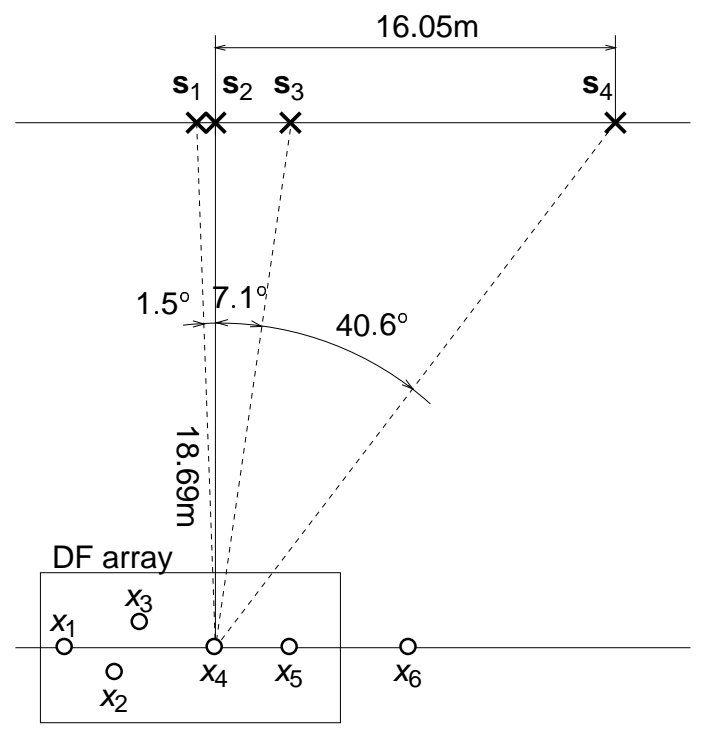

Fig. 9. Experimental set-up, with $4 \mathrm{FM}$ transmitters $\left(\mathbf{s}_{1}, \cdots, \mathbf{s}_{4}\right)$ and an antenna array consisting of 6 receivers

noise level) is seen in the singular values of $\hat{P}$ as an increase in the small singular values, which will limit the detection at some point. The average modulus error of a $5 \mathrm{~dB}$ signal is expected to be 0.20 , which matches with the figures.

The angular spacing between the first two signals can be reduced to $10^{\circ}$ without problems. When the spacing is further reduced to $5^{\circ}$ (figure 8 ), the detection of the two closely spaced $\mathrm{CM}$ signals becomes problematic, and for $n=26$, GSA postprocessing failed to keep two of the signals independent. The reason is that, because of the close angles of arrival, the condition number of the $A$-matrix is increased (from 2.3 to 11.9), which, at this noise level, is sufficient to close the gap between the large and small singular values for any $n$. This is confirmed by theoretical predictions of the gap size (section V-C). Nonetheless, $n=100$ was sufficient in simulations to separate the signals even without additional GSA iterations: the loss of gap prevents detection of the number of CM signals, but not necessarily their separation.

\section{B. Experiments on measured data}

The algorithm was also tested on data collected from an experimental roof-top antenna array. ${ }^{7}$ The configuration of the array is shown in figure 9 . The receiving array consisted of $m=6$ isotropic antennas, where antennas $x_{1}-x_{5}$ formed part of an airplane DF array with a baseline of approximately $1.5 \mathrm{~m}$, and antenna $x_{6}$ was a dipole at approximately $1 \mathrm{~m}$ to the right of the array. Located nearby were $d=4$ dipole antennas, marked $\mathbf{s}_{1}-$ $\mathbf{s}_{4}$, each broadcasting FM signals at RF carrier frequencies of $902.1 \mathrm{MHz} \pm 200 \mathrm{~Hz}$ (i.e., the individual carrier frequencies were slightly offset). The signal transmitted by source $\mathbf{s}_{1}$ was an FM-modulated tone of $1 \mathrm{kHz}$, signals $\mathbf{s}_{2}-\mathbf{s}_{4}$ consisted of FM modulated speech and music. The received signals were RFdemodulated, sampled at $37.5 \mathrm{kHz}$ (complex), digitized at 12 bits, and band-limited at $25 \mathrm{kHz}$. The actual $10 \mathrm{~dB}$ bandwidth of the sources was around $6 \mathrm{kHz}$. In the first experiment, the power of each transmitted signal with respect to the ambient background noise (SNR) was $19.1 \mathrm{~dB}, 17.6 \mathrm{~dB}, 17.9 \mathrm{~dB}, 16.7$ $\mathrm{dB}$, respectively. In a second experiment, the power of $\mathbf{s}_{2}$ was lowered to $\operatorname{SNR}\left(\mathbf{s}_{2}\right)=7.6 \mathrm{~dB}$.

In figure 10, the singular values of $X$ and $\hat{P}$ are shown. For $n=100$ and $n=50$, it is clear that there are four CM signals. (For the record, we mention that the condition number of $A$ was later estimated as 5.8.) Denote by $\hat{d}, \hat{\delta}$ the parameters used by the ACMA, as opposed to the true values $(d=\delta=4)$. Figure 11 shows the modulus error during subsequent Gerchberg iterations when the ACMA is run with $\hat{d}, \hat{\delta}=4$. For $n=50$, the analytically computed values of $\mathbf{w}$ are hardly changed; for $n=17$, the Gerchberg iterations improve a bit on the w. With random initializations, the Gerchberg iterations may converge to at least two spurious local minima.

Table II lists the estimated signal-to-interference ratios (SIRs) obtained by the ACMA, both before and after the additional GSA iterations. The values are based on the rows of the matrix $\hat{W} A$, where $\hat{W}$ contains the weight vectors as determined by the algorithm for the listed $n, \hat{d}, \hat{\delta}$, and $A$ is an estimate of the unknown true $A$ matrix computed using the ACMA on $n=400$ samples and with $\hat{d}=\hat{\delta}=4$. $\hat{W} A$ should be close to the identity matrix (or a permutation and diagonal scaling thereof). The table shows the results obtained for various choices of the parameters $\hat{d}$ and $\hat{\delta}$ used in the algorithm. It is seen that overestimating $d$ is not re-

\footnotetext{
${ }^{7}$ The data was measured and provided by ARGOSystems, Inc., Sunnyvale, $\mathrm{CA}$, as part of an ongoing research project with Stanford University.
} 
(a)

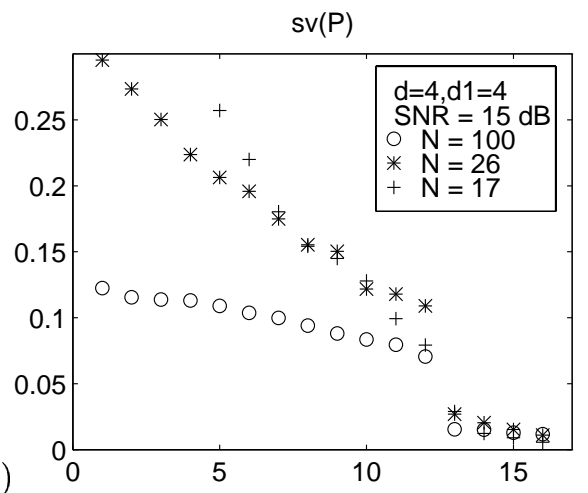

(b)

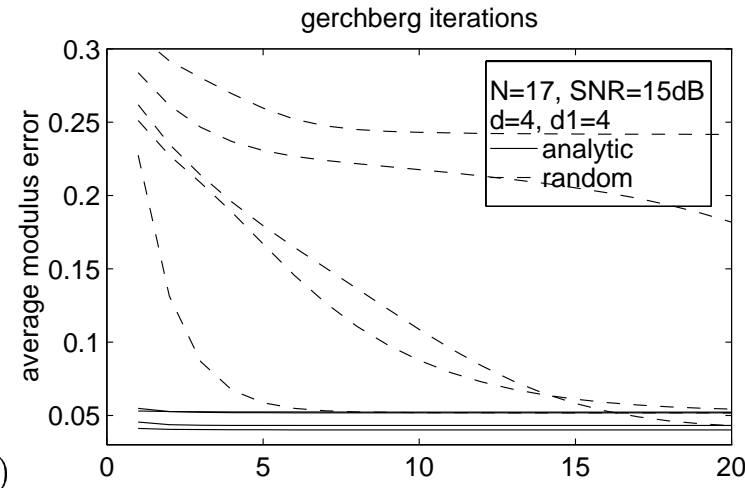

Fig. 4. $4 \mathrm{CM}$ signals. Each signal has $\mathrm{SNR}=15 \mathrm{~dB}, \theta_{2}=30^{\circ}$. (a) Singular values of $\hat{P}$. (b) Convergence of Gerchberg algorithm for analytically computed initial points, and for random initial points, for $n=17$.

$\operatorname{sv}(\mathrm{P})$

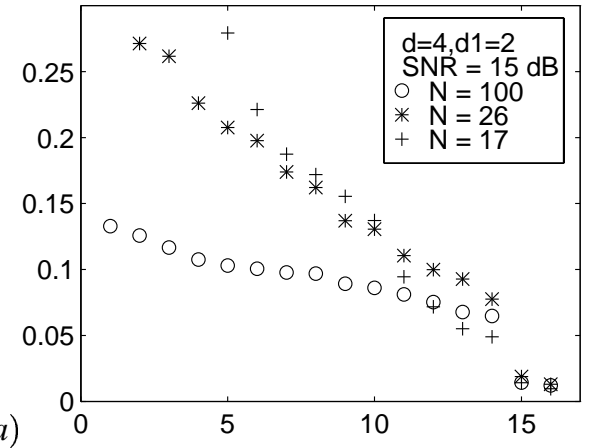

(b)

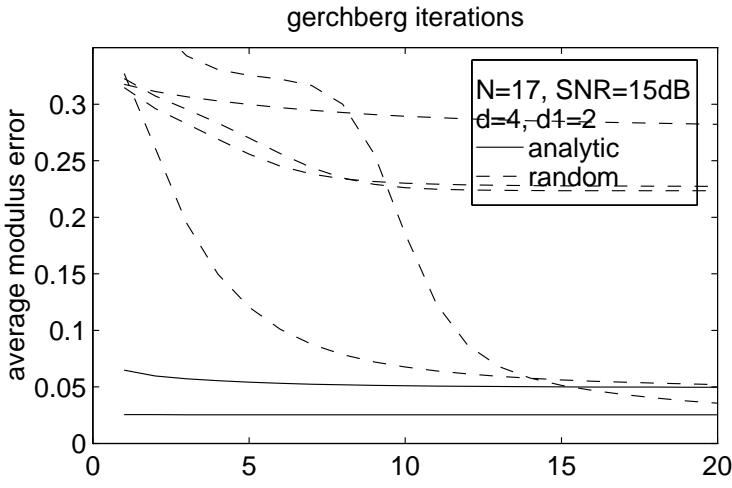

Fig. 5. 4 signals, $2 \mathrm{CM}$ signals. Each signal has $\mathrm{SNR}=15 \mathrm{~dB}, \theta_{2}=30^{\circ}$.

$\operatorname{sv}(\mathrm{P})$

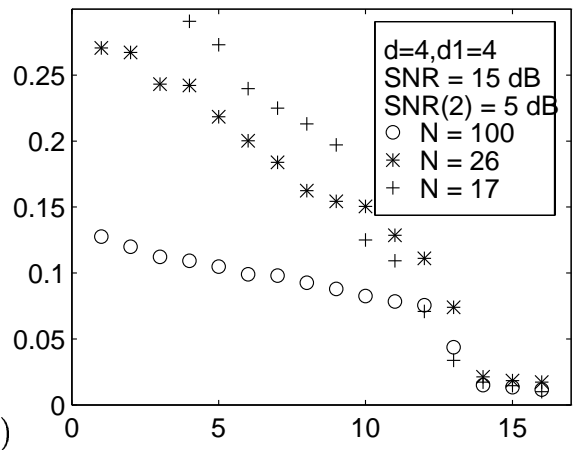

(b)

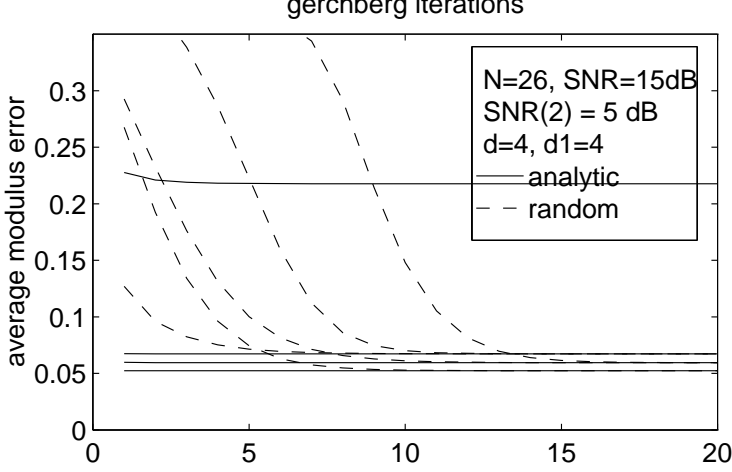

Fig. 6. $4 \mathrm{CM}$ signals. Signal 2 has $\mathrm{SNR}=5 \mathrm{~dB}, \theta_{2}=30^{\circ}$; the other signals have $\mathrm{SNR}=15 \mathrm{~dB}$. $\operatorname{sv}(P)$

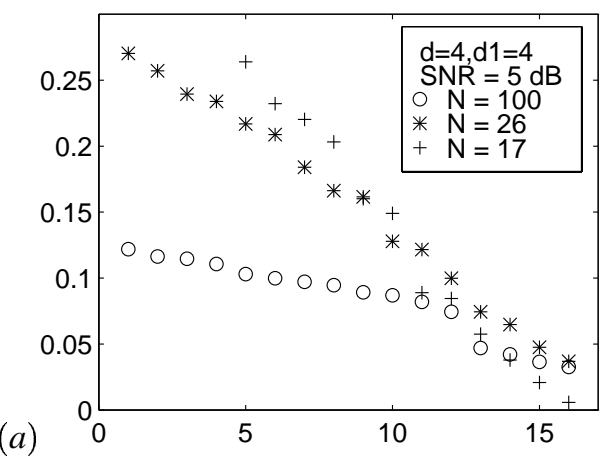

(b)

gerchberg iterations

Fig. 7. $4 \mathrm{CM}$ signals. Each signal has $\mathrm{SNR}=5 \mathrm{~dB}, \theta_{2}=30^{\circ}$. 

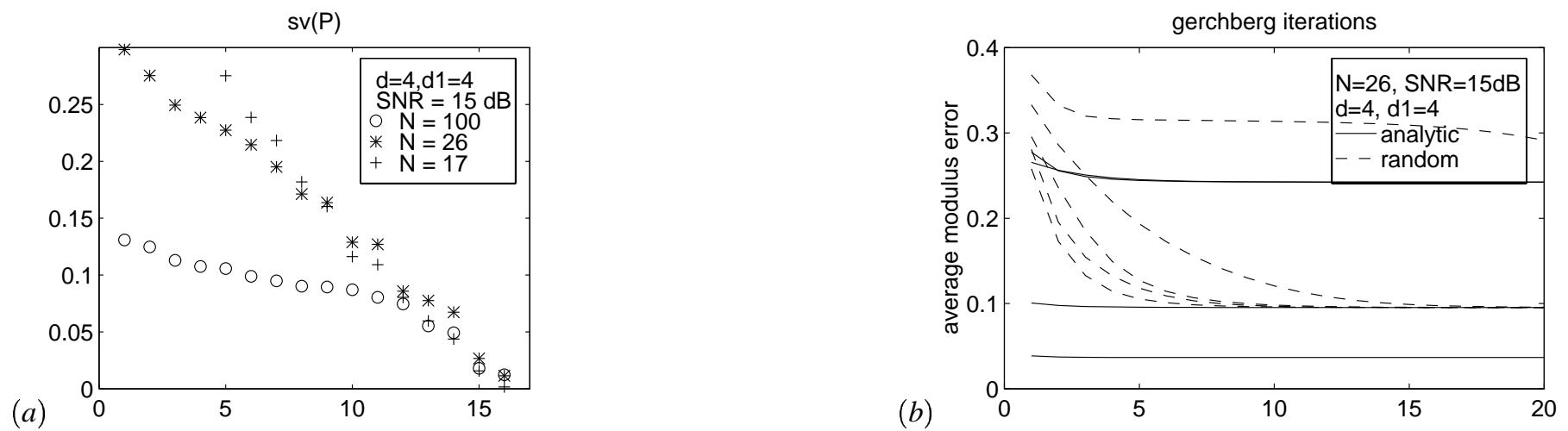

Fig. 8. 4 CM signals. Each signal has $\mathrm{SNR}=15 \mathrm{~dB}, \theta_{2}=5^{\circ}$.

(a)

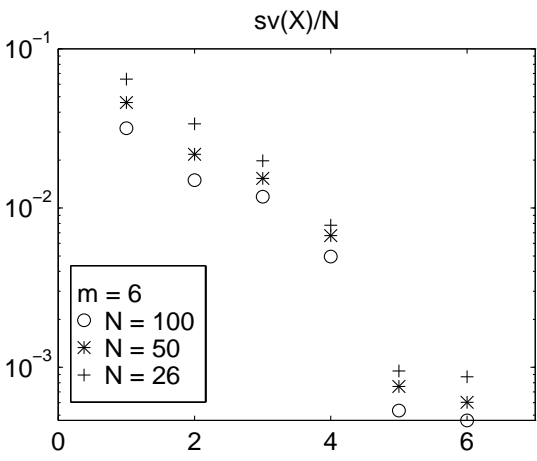

Fig. 10. Experiment with 4 FM signals and 6 antennas; SNR $\left(\mathbf{s}_{2}\right)=17.6 \mathrm{~dB}$. (a) singular values of $X ;(b)$ singular values of $\hat{P}$, with $\hat{d}=4$. gerchberg iterations

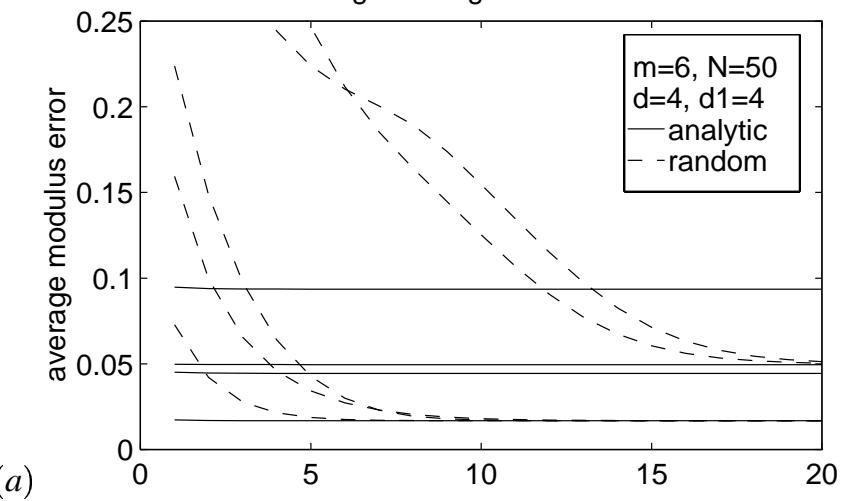

Fig. 11. Gerchberg iterations, for $\hat{d}=4, \hat{\delta}=4 ; \operatorname{SNR}\left(\mathbf{s}_{2}\right)=17.6 \mathrm{~dB}$. (a) $n=50,(b) n=17$.
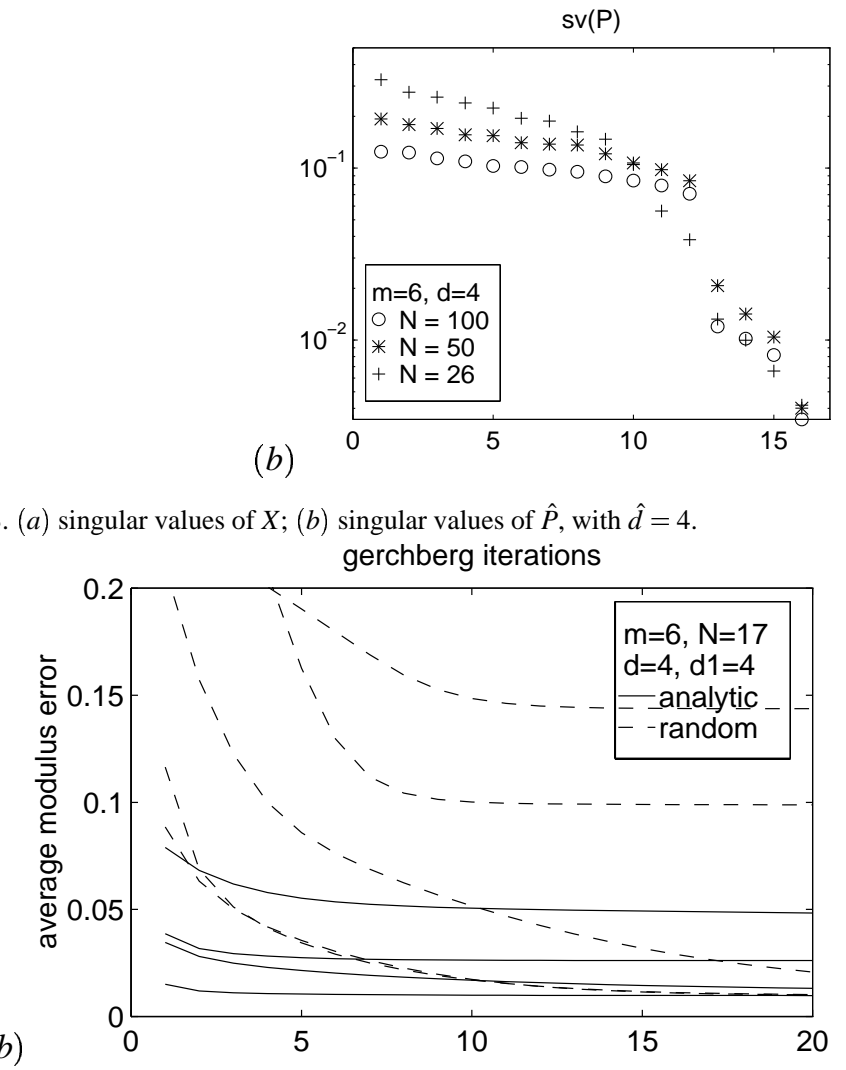

gerchberg iterations

$(b)$ 
TABLE II

WORST SIR [dB] AFTER SEPARATION, CASE SNR $\left(\mathbf{s}_{2}\right)=17.6 \mathrm{~dB}$ (WORST RECEIVED SIR $=-2.4 \mathrm{~dB} /$ ANTENNA)

\begin{tabular}{rrr|rr|rr|rr}
\hline \multicolumn{4}{c}{$\hat{d}=4, \hat{\delta}=4$} & \multicolumn{2}{c}{$\hat{d}=5, \hat{\delta}=4$} & \multicolumn{2}{c}{$\hat{d}=6, \hat{\delta}=4$} & \multicolumn{2}{c}{$=5, \hat{\delta}=5$} \\
\hline \multicolumn{2}{r|}{ ACMA } & +GSA & ACMA & +GSA & ACMA & +GSA & ACMA & +GSA \\
\hline$n=100$ & 36.0 & 34.8 & 35.4 & 34.8 & 35.1 & 34.8 & 36.0 & 34.8 \\
50 & 27.2 & 26.8 & 23.5 & 26.9 & 19.5 & 26.9 & $(18.9)$ & $(36.9)$ \\
26 & 12.6 & 22.8 & 6.6 & 25.1 & & & 3.0 & 25.3 \\
17 & 8.2 & 17.2 & & & & & & \\
\hline
\end{tabular}

$(\cdot)$ : not all signals were recovered

TABLE III

WORST SIR [dB] AFTER SEPARATION, CASE SNR $\left(\mathbf{s}_{2}\right)=7.6 \mathrm{~dB}$ (WORST RECEIVED SIR $=-11.5 \mathrm{~dB} /$ ANTENNA)

\begin{tabular}{|c|c|c|c|c|c|c|c|c|}
\hline & \multicolumn{2}{|c|}{$\hat{d}=4, \hat{\delta}=4$} & \multicolumn{2}{|c|}{$\hat{d}=5, \hat{\delta}=4$} & \multicolumn{2}{|c|}{$\hat{d}=5, \hat{\delta}=5$} & \multicolumn{2}{|c|}{$\hat{d}=3, \hat{\delta}=3$} \\
\hline & $\overline{\mathrm{ACMA}}$ & $+\mathrm{GSA}$ & ACMA & $+\mathrm{GSA}$ & ACMA & $+\mathrm{GSA}$ & ACMA & $+\mathrm{GSA}$ \\
\hline$n=100$ & 24.0 & 23.1 & 22.5 & 23.1 & 22.4 & 23.1 & (14.1) & $(34.9)$ \\
\hline 50 & 12.2 & 18.6 & 9.5 & 18.5 & 14.4 & 18.4 & (14.2) & $(33.2)$ \\
\hline 26 & 0.0 & 2.3 & -3.8 & 9.4 & $(8.2)$ & (19.6) & (12.7) & $(26.1)$ \\
\hline 17 & $(7.0)$ & (9.5) & & & & & (12.4) & (12.8) \\
\hline
\end{tabular}

$(\cdot)$ : not all signals were recovered

(a)
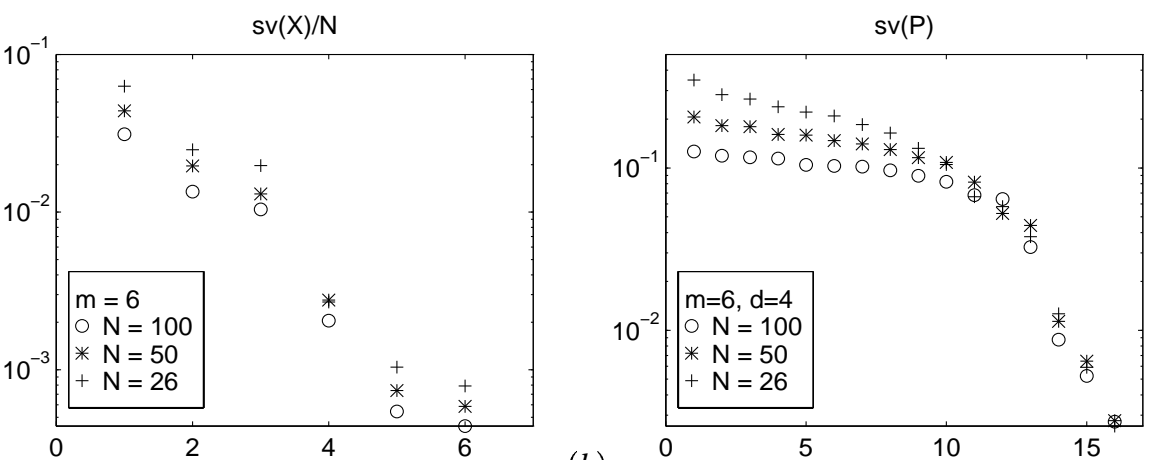

(b)

(c)

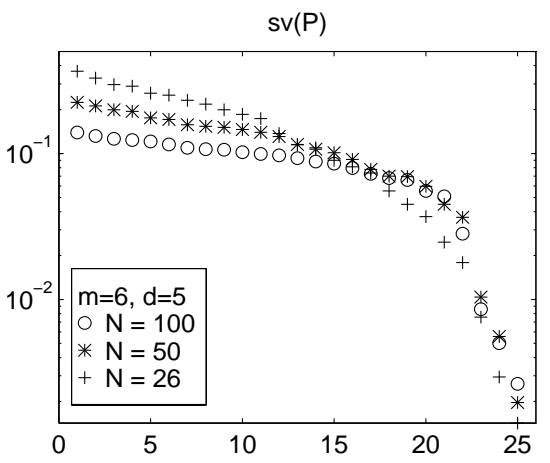

Fig. 12. Experiment with 4 FM signals; $\operatorname{SNR}\left(\mathbf{s}_{2}\right)=7.6 \mathrm{~dB}$. (a) singular values of $X ;(b)$ singular values of $\hat{P}$, with $\hat{d}=4$, and $(c)$ with $\hat{d}=5$.

(a)

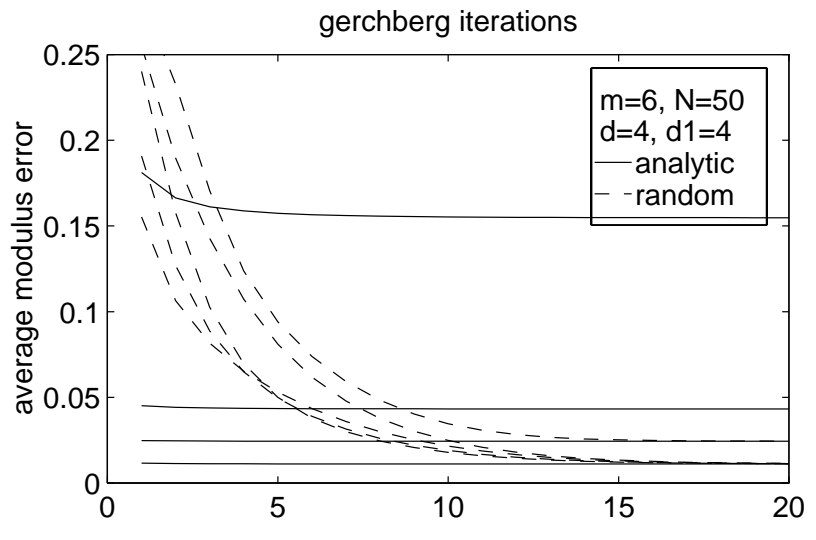

(b)

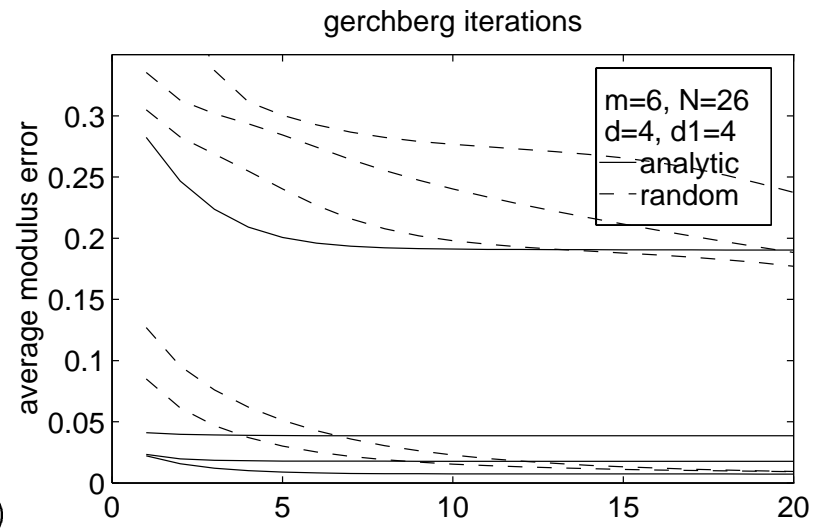

Fig. 13. Gerchberg iterations, for $\hat{d}=4, \hat{\delta}=4 ; \operatorname{SNR}\left(\mathbf{s}_{2}\right)=7.6 \mathrm{~dB}$. (a) $n=50,(b) n=26$. 
$\mathbf{s}_{2}$ is of course lost, but the behavior of the other estimates is approximately the same.

Note from the tables that the SIR is not always improved by the additional Gerchberg iterations. The reason is that the GSA is connected to a different cost function, (29) rather than (17), with slightly different minima for finite $n$ and SNR. None of these minima are necessarily coinciding with minimal SIR.

The main conclusion to draw from the experiments carried out in this section is that in all observed cases the algorithm obtains the optimum of the minimization problem if $n$ is sufficiently large. For four signals, $n=50-100$ is typically large enough, even under severe conditions. For smaller $n$, the estimates move away from their optimal values, but usually, the algorithm still finds all CM signals if their number has been estimated correctly, and the optima can be obtained by adding a few iterations of the Gerchberg-Saxton algorithm as postprocessing. The effect of a smaller $n$ is mostly felt in a closing of the gap between the larger and smaller singular values of $\hat{P}$, which limits the detection of $\delta$. This is mitigated to some extent by the property that the algorithm is quite robust when $d$ or $\delta$ are overestimated.

\section{Detection thresholds}

What determines the singular values of $\hat{P}$, and thus the resolution of the algorithm? This is the topic of a separate paper, but it is relevant to at least summarize some of the results here, as they explain some properties of the singular value plots quantitatively.

The large singular values of $\hat{P}$ tend towards $1 / \sqrt{n}$, but for small values of $n$, they are not constant yet but distributed along a line. This distribution is similar to that of the singular values of a random matrix, which has been investigated in [53,54]. Extrapolating the result in [53], the smallest among the set of $d^{2}-d$ large singular values is (with probability better than 0.95 ) expected to satisfy

$$
\min \left(\text { large sv) }>\frac{1}{\sqrt{n}}-\frac{d-\frac{1}{2}}{n} .\right.
$$

This matches with the experiments earlier in this section as well. At the other side of the gap, the $\delta$ small singular values of the numerical kernel should ideally be equal to 0 , but with noise they are increased to

$$
\max (\text { small sv })=\frac{\sigma \sqrt{2}}{\sqrt{n m}} \cdot \operatorname{cond}(A) .
$$

Here, $\sigma^{2}$ is the normalized noise power per sample per antenna: $20 \log (1 / \sigma)$ is the SNR of the strongest signal at a single receiver. The noise is enhanced by a factor $\sqrt{2}$ because of the inherent squaring of the data. The factor " $\operatorname{cond}(A)$ " is the conditioning of $A$, and includes two effects. When the array response is approximately uniform in all directions, $\operatorname{cond}(A)$ is just the square root of the ratio of the power of the strongest signal to the weakest: this translates $\sigma$ into the SNR of the weakest signal. A large (bad) condition number of $A$ may also be due to a close spacing of two signals, as determined by the resolution limit of the array. In such cases, a correction by $1 / \sqrt{2}$ is sometimes in order. The above two equations allow to derive the maximal noise power for which there still can be a gap as

$$
\mathrm{SNR}>3 \mathrm{~dB}-10 \log m+20 \log \operatorname{cond}(A),
$$

(independent of $n$ ), and an indication of the minimal number of samples that is needed in that case,

$$
n>\left[\frac{d-\frac{1}{2}}{1-\sigma \sqrt{2} \operatorname{cond}(A) / \sqrt{m}}\right]^{2}
$$

(we still require $n>d^{2}$, too). They also allow to set automatic decision thresholds for rank detection in subspace estimators.

\section{CONCLUDING REMARKS}

In this paper, we have described an analytic method for solving the constant modulus problem. The method condenses all conditions on the weight vectors $\mathbf{w}$ into a single matrix $\hat{P}$, and finds all independent vectors in the kernel of this matrix that have a Kronecker product structure. This problem, in turn, is shown to be a generalized matrix pencil (eigenvalue) problem, which may be formulated in terms of a super-generalized Schur decomposition: for given matrices $Y_{1}, \cdots, Y_{\delta}$, find $Q, Z$ (unitary) such that

$$
\begin{aligned}
Q Y_{1} Z & =R_{1} \\
Q Y_{\delta} Z & \vdots \\
& R_{\delta}
\end{aligned}
$$

where $R_{1}, \cdots, R_{\delta}$ are as much upper triangular as possible. We have proposed a modified QZ iteration which treats all $Y_{k}$ equally, converges to upper triangular matrices $R_{k}$ in the absence of noise, and usually has quadratic convergence in our simulations.

The analytic algorithm is definitely more complex to implement than the usual iterative approaches for blind beamforming and blind deconvolution of constant modulus signals. However, it gives fundamental solutions to a number of problems that have plagued iterative $\mathrm{CM}$ algorithms ever since their inception in the early 1980s. The most important advantages of the analytic approach are

1. It is less blind: the number of CM signals are detected explicitly from the close-to-zero singular values of $\hat{P}$. Not all signals have to be $\mathrm{CM}$ signals.

2. It is deterministic: the minima of the cost functions are found by analysis, rather than by trying different initial points in the usual steepest gradient methods. The only parameters that have to be set are the total number of signals, and the number of CM signals.

3. It is robust on small data sets and in the presence of noise, although a few additional iterations of the standard CMA may be necessary to find the optimal weight vectors.

4. There are detection criteria that predict how many antennas and samples are needed in given scenarios (cf. section V-C). The modest requirements on the number of samples is an important issue in applications where multipath causes fast fading.

Signals that are not CM signals but have a kurtosis ${ }^{8}$ smaller than two, e.g., QAM and other finite alphabet signals, may be modeled as a CM signal at the RMS amplitude plus a limited amount of noise corresponding to the variance on this amplitude. When the equivalent noise power satisfies (32), then the number of samples $n$ can be chosen large enough to allow detection of the signal as a CM signal, and thus to recover this signal as

\footnotetext{
${ }^{8}$ The kurtosis of a signal $x(t)$ is defined as $\mathrm{\kappa}(x)=\mathrm{E}|x(t)|^{4} /\left(\mathrm{E}|x(t)|^{2}\right)^{2}$.
} 
well. This "abuse" of the CM property to separate independent non-Gaussian signals is of course already common practice in blind equalizers and beamformers ever since their invention (viz. $[6,55])$. It might even be argued that the fourth-order cumulant techniques in [15-17], constructed to separate independent nonGaussian signals, do in fact rely on the same property. Further research is needed to bring the many hidden connections into perspective.

\section{Acknowledgements}

We are grateful to Frank McCarthy of ARGOSystems, Inc., for sharing his measurement data reported on in section V-B. The first author would like to thank Prof. Gene Golub for inviting him to Stanford University, and the anonymous reviewers for their stimulating critiques.

\section{APPENDICES}

\section{VECTORING OF HERMITIAN MATRICES}

For hermitian matrices $Y$, we can redefine the vectoring operation $\operatorname{vec}(Y)$ to take advantage of the symmetry in $Y$, and end up with about half the number of parameters. One convenient way to do so leads to real vectors instead of complex vectors and is based on the property (for $x \in \mathbb{C}$ )

$$
\sqrt{2}\left[\begin{array}{l}
\operatorname{Re}(x) \\
\operatorname{Im}(x)
\end{array}\right]=\frac{1}{\sqrt{2}}\left[\begin{array}{cc}
1 & 1 \\
-j & j
\end{array}\right]\left[\begin{array}{c}
x \\
x^{*}
\end{array}\right] .
$$

Hence, a unitary matrix will transform a vector in which both $x$ and $x^{*}$ are present into a vector where these components are real. Thus define, for hermitian matrices $Y \in \mathbb{C}^{d \times d}$, the hermitian vectoring operation "vech" as

$$
\underline{\mathbf{y}}=\operatorname{vech}(Y)=\left[\begin{array}{c}
Y_{11} \\
\operatorname{Re}\left(Y_{12}\right) \sqrt{2} \\
\operatorname{Re}\left(Y_{13}\right) \sqrt{2} \\
\vdots \\
\operatorname{Im}\left(Y_{12}\right) \sqrt{2} \\
Y_{22} \\
\vdots \\
Y_{d d}
\end{array}\right]
$$

i.e.,

$$
\underline{\mathbf{y}}_{(i-1) d+k}=\left\{\begin{array}{ll}
Y_{i i}, & i=k \\
\operatorname{Re}\left(Y_{i k}\right) \sqrt{2}, & i<k \\
\operatorname{Im}\left(Y_{k i}\right) \sqrt{2}, & i>k
\end{array} \quad i, k=1, \cdots, d .\right.
$$

There is a unitary matrix ( $U$, say) with a simple structure, mapping the result of the original vectoring operation into the new result: $\underline{\mathbf{y}}=U \operatorname{vec}(Y)$. The inverse operation is $Y=\operatorname{vec}^{-1}\left(U^{*} \underline{\mathbf{y}}\right)=$ : $\operatorname{vech}^{-1}(\underline{\mathbf{y}})$, which also may be evaluated explicitly.

Besides the fact that $\operatorname{vech}(\cdot)$ is a real vector, a second advantage is that the inverse operation vech ${ }^{-1}$ returns matrices that are hermitian by construction. Both advantages show up when we elaborate on our application (equation (7)). Because $P_{k}=\mathbf{v}_{k} \mathbf{v}_{k}^{*}$ is hermitian, $\underline{\mathbf{p}}_{k}$ is a real vector. The implication is that the matrix $\underline{P}$, constructed from these vectors, is real, as is the matrix $\underline{\hat{P}}$. Hence, the SVD of $\underline{\hat{P}}$ is a real SVD, which saves about a factor of 3 on computations. Because of the unitarity of the transformation, the singular values of $\hat{P}$ and $\underline{\hat{P}}$ are precisely the same, but the basis $\left\{\underline{\mathbf{y}}_{k}\right\}$ that we select from the kernel of $\underline{\hat{P}}$ consists of real vectors. As a result, the matrices $Y_{1}=\operatorname{vech}^{-1}\left(\underline{\mathbf{y}}_{1}\right), \cdots, Y_{\delta}=$ $\operatorname{vech}^{-1}\left(\underline{\mathbf{y}}_{\delta}\right)$ that we form from the basis are hermitian by construction.

\section{PROOFS}

\section{Proof of lemma 1}

Without loss of generality we may take $d=m$. Our approach is to determine how many vectors $\mathbf{w}$ there can be such that $\mathbf{w} X$ is a CM signal. As derived in section II-C, each column of $X$ gives a quadratic equation that the entries of $\mathbf{w}$ have to satisfy. We assume that these constraints are independent.

Since $\mathbf{w}$ is a complex vector, it consists of $2 d$ parameters. Any w can be scaled by a unimodular scaling such that its first entry is real and positive; since this scaling does not affect the constantmodulus property of $\mathbf{w} X$, it is an unconstrained parameter, so that the $n$ columns of $X$ only put constraints on the remaining $2 d-1$ parameters. On general principles, we expect that when $n=2 d-1$, there is only a discrete set of isolated solutions for w. Nonetheless, this set might be too large: e.g., when $d=2$, the isolated solutions are determined by the intersection of 3 ellipsoids in 3-space, with 0,2 or 4 solutions. Adding one more constraint (i.e., $n=2 d$ ) will place a new condition on the isolated solutions, to which in general only the original CM source signals and their weight vectors can comply.

\section{Proof of lemma 3}

To prove equivalence, it remains to show that a set of solutions $\left\{\mathbf{y}_{k}\right\}_{1}^{\delta}$ of the form $\mathbf{y}_{k}=\mathbf{w}_{k} \otimes \overline{\mathbf{w}}_{k}$ is linearly independent if and only if the corresponding set $\left\{\mathbf{w}_{k}\right\}_{1}^{\delta}$ is linearly independent. Indeed, with $Y_{k}=\mathbf{w}_{k}^{*} \mathbf{w}_{k}$ and $\mathbf{y}_{k}=\mathbf{w}_{k} \otimes \overline{\mathbf{w}}_{k}=\operatorname{vec}\left(Y_{k}\right)$,

$$
\begin{aligned}
& \left\{\mathbf{y}_{k}\right\}_{1}^{\delta} \text { is a linearly independent set } \\
& \Leftrightarrow\left[\alpha_{1} \mathbf{y}_{1}+\alpha_{2} \mathbf{y}_{2}+\cdots+\alpha_{\delta} \mathbf{y}_{\delta}=0 \Rightarrow \alpha_{i}=0, i=1, \cdots, \delta\right] \\
& \Leftrightarrow\left[\alpha_{1} Y_{1}+\alpha_{2} Y_{2}+\cdots+\alpha_{\delta} Y_{\delta}=0 \Rightarrow \alpha_{i}=0, i=1, \cdots, \delta\right] \\
& \Leftrightarrow \operatorname{rank}\left[Y_{1} \quad Y_{2} \cdots Y_{\delta}\right]=\delta \\
& \Leftrightarrow \operatorname{rank}\left[\mathbf{w}_{1}^{*} \quad \mathbf{w}_{2}^{*} \cdots \mathbf{w}_{\delta}^{*}\right]=\delta \\
& \Leftrightarrow\left\{\mathbf{w}_{k}\right\}_{1}^{\delta} \text { is a linearly independent set. }
\end{aligned}
$$

\section{Proof of lemma 4}

The only issue to show is the equivalence of $\hat{\mathbf{p}}_{1} \mathbf{y}=n^{1 / 2}$ to $\|\mathbf{w}\|=n^{1 / 2}$. This proof consists of two (technical) steps.

1. We first show that $\hat{\mathbf{p}}_{1} \mathbf{y}=n^{1 / 2} \Leftrightarrow \operatorname{tr}(Y)=n$, where $Y=$ $\operatorname{vec}^{-1} \mathbf{y} . \quad\left(\operatorname{tr}(\cdot)\right.$ is the trace operator.) Indeed, let $\hat{P}_{1}=$ $\operatorname{vec}^{-1}\left(\hat{\mathbf{p}}_{1}\right)$. We show that $\hat{P}_{1}=n^{-1 / 2} I$. For this, we use the fact that $Q$ is unitary and $P$ is constructed from $\hat{V}$, an isometry. $\hat{\mathbf{p}}_{1}$ only depends on the first row of $Q$. This row must be equal to $n^{-1 / 2}[1 \cdots 1]$, because all other rows of $Q$ are necessarily orthogonal to this vector. Using the definition of $P$ gives

$$
n^{1 / 2} \hat{P}_{1}=\mathbf{v}_{1} \mathbf{v}_{1}^{*}+\cdots+\mathbf{v}_{n} \mathbf{v}_{n}^{*}=\hat{V} \hat{V}^{*}=I .
$$


Finally, it remains to note that $\hat{\mathbf{p}}_{1} \mathbf{y}=\mathbf{w} \hat{P}_{1} \mathbf{w}^{*}=\mathbf{w w}^{*} n^{-1 / 2}=$ $\operatorname{tr}\left(\mathbf{w}^{*} \mathbf{w}\right) n^{-1 / 2}=\operatorname{tr}(Y) n^{-1 / 2}$.

2. Furthermore, when $\mathbf{y}=\mathbf{w} \otimes \overline{\mathbf{w}}$, then $\operatorname{tr}(Y)=\operatorname{tr}\left(\mathbf{w}^{*} \mathbf{w}\right)=$ $\|\mathbf{w}\|^{2}$ so that $\hat{\mathbf{p}}_{1} \mathbf{y}=n^{1 / 2} \Leftrightarrow\|\mathbf{w}\|^{2}=n$.

\section{Proof of proposition 5}

The relation between $\hat{V}$ and $S$ may be written as $\hat{V}=\hat{A} S$, where the nonsingular $d \times d$ matrix $\hat{A}$ is derived from the original antenna response matrix. $P$ was defined in terms of $\hat{V}$ in (8); in a similar way, we may define a matrix $P_{S}$ in terms of $S$. This produces

$$
P=P_{S} \cdot\left[\hat{A}^{T} \otimes \hat{A}^{*}\right], \quad P_{S}=\left[\begin{array}{c}
{\left[\overline{\mathbf{s}}_{1} \otimes \mathbf{s}_{1}\right]^{T}} \\
\vdots \\
{\left[\overline{\mathbf{s}}_{n} \otimes \mathbf{s}_{n}\right]^{T}}
\end{array}\right]
$$

The $d^{2} \times d^{2}$ matrix $\left[\hat{A}^{T} \otimes \hat{A}^{*}\right]$ is nonsingular: its singular values are given by all cross-products of the singular values of $\hat{A}$. Hence the propagation environment does not influence the dimension of the kernel of $P$ (or $\hat{P}$ ): it will be too large only if there are specific phase relations between the signals, valid for all points in time. It is not a trivial task to analyze these relations, except for the case $d=\delta=2$, which is done in the main text. For statistically independent signals with a rich enough phase space (analog FM or PM signals, or digital CM signals with reasonably large constellations or sufficient oversampling), the probability is zero that the rank of the matrix $P_{S}$ is any lower than necessary. This becomes more so for larger $n$.

\section{Proof of proposition 7}

$\overline{\mathbf{w}}) \|^{2}$

We first show that $\operatorname{dist}(\mathbf{w} \hat{V}, \mathcal{C M})=n^{-1}\left(n-\mathbf{w w}^{*}\right)^{2}+\| \hat{P}(\mathbf{w} \otimes$

Indeed, take the definition of $\operatorname{dist}(\cdot, \mathcal{C M})$ in equation (17), and make the same series of substitutions as in section II-C:

$$
\begin{aligned}
\operatorname{dist}(\mathbf{w} \hat{V}, \mathcal{C M}) & =\sum\left(\left|\mathbf{w} \mathbf{v}_{k}\right|^{2}-1\right)^{2} \\
& =\sum\left(1-\mathbf{w} P_{k} \mathbf{w}^{*}\right)^{2} \\
& =\sum\left(1-\mathbf{p}_{k} \mathbf{y}\right)^{2} \quad\left(\mathbf{y}=\mathbf{w} \otimes \mathbf{w}^{*}\right) \\
& =\left\|\left[\begin{array}{c}
1 \\
\vdots \\
1
\end{array}\right]-P \mathbf{y}\right\|^{2} \\
& =\left\|\left[\begin{array}{c}
n^{1 / 2} \\
0
\end{array}\right]-\left[\begin{array}{c}
\hat{\mathbf{p}}_{1} \\
\hat{P}
\end{array}\right] \mathbf{y}\right\|^{2} \\
& =n^{-1}\left(n-\mathbf{w} \mathbf{w}^{*}\right)^{2}+\|\hat{P} \mathbf{y}\|^{2} .
\end{aligned}
$$

In making the last step, we have used the proof of lemma 4 : $n^{1 / 2}-\hat{\mathbf{p}}_{1} \mathbf{y}=n^{-1 / 2}\left(n-\mathbf{w w}^{*}\right)$.

Hence,

we

have shown that the distance function $\operatorname{dist}(\mathbf{w} \hat{V}, \mathcal{C M})$ splits into two terms. The first term $n^{-1}\left(n-\mathbf{w w}^{*}\right)^{2}$ is only a penalty on the norm of $\mathbf{w}:\|\mathbf{w}\|$ should be close to $n^{1 / 2}$. A multiplication of $\mathbf{w}$ by some number $c$ will scale both $\left(\mathbf{w} \mathbf{w}^{*}\right)^{2}$ and $\|\hat{P}(\mathbf{w} \otimes \overline{\mathbf{w}})\|$ by $c^{2}$. This means that the given minimization problem is separable into the constrained minimization problem for $\mathbf{w}$,

$$
\varepsilon^{2}:=\min \|\hat{P}(\mathbf{w} \otimes \overline{\mathbf{w}})\|^{2} \quad \text { s.t. } \mathbf{w w}^{*}=n,
$$

which will provide the direction of $\mathbf{w}$, and the computation of a scalar $c$ to minimize $\operatorname{dist}(c \mathbf{w} \hat{V}, \mathcal{C M})$. After solving the first problem, the optimal value for $c$ is directly determined by $\min _{c} n^{-1}\left(n-c^{2} n\right)^{2}+c^{4} \varepsilon^{2}$, which has the solution

$$
c^{2}=\frac{n}{n+\varepsilon^{2}} \text {. }
$$

\section{REFERENCES}

[1] A.J. van der Veen and A. Paulraj, "A constant modulus factorization technique for smart antenna applications in mobile communications," in Proc. SPIE, "Advanced Signal Processing Algorithms, Architectures, and Implementations V" (F.T. Luk, ed.), vol. 2296, (San Diego, CA), pp. 230-241, July 1994

[2] A.J. van der Veen, S. Talwar, and A. Paulraj, "Blind estimation of multiple digital signals transmitted over FIR channels," IEEE Signal Processing Letters, vol. 2, pp. 99-102, May 1995.

[3] R.O. Schmidt, "Multiple emitter location and signal parameters estimation," IEEE Trans. Antennas and Prop., vol. 34, pp. 276-280, Mar. 1986.

[4] R. Roy, A. Paulraj, and T. Kailath, "ESPRIT-a subspace rotation approach to estimation of parameters of cisoids in noise," IEEE Trans. Acoust., Speech, Signal Processing, vol. 34, no. 5, pp. 1340-1342, 1986.

[5] Y. Sato, "A method of self-recovering equalization for multilevel amplitude-modulation systems," IEEE Trans. Communications, vol. 23, pp. 679-682, June 1975.

[6] D.N. Godard, "Self-recovering equalization and carrier tracking in twodimensional data communication systems," IEEE Trans. Communications, vol. 28, pp. 1867-1875, Nov. 1980.

[7] J.R. Treichler and B.G. Agee, "A new approach to multipath correction of constant modulus signals," IEEE Trans. Acoust., Speech, Signal Processing, vol. 31, pp. 459-471, Apr. 1983.

[8] M.G. Larimore and J.R. Treichler, "Convergence behavior of the constant modulus algorithm," in Proc. IEEE ICASSP, pp. 13-16 vol.1, 1983.

[9] S. Haykin, ed., Blind Deconvolution. Englewood Cliffs: Prentice Hall, 1994.

[10] J.R. Treichler and M.G. Larimore, "New processing techniques based on constant modulus adaptive algorithm," IEEE Trans. Acoust., Speech, Signal Processing, vol. 33, pp. 420-431, Apr. 1985.

[11] R. Gooch and J. Lundell, "The CM array: An adaptive beamformer for constant modulus signals," in Proc. IEEE ICASSP, (Tokyo), pp. 2523-2526, 1986.

[12] R.P. Gooch and B.J. Sublett, "Joint spatial and temporal equalization in a decision-directed adaptive antenna system," in 22-nd Asilomar Conf. on Signals, Systems and Computers, pp. 255-259 vol.1, Maple Press, 1988.

[13] S. Talwar, M. Viberg, and A. Paulraj, "Blind estimation of multiple cochannel digital signals arriving at an antenna array," in 27-th Asilomar Conf. Signals, Syst. Comp., pp. 349-353 vol.1, IEEE, 1993.

[14] B.G. Agee, S.V. Schell, and W.A. Gardner, "Spectral self-coherence restoral: A new approach to blind adaptive signal extraction using antenna arrays," Proc. IEEE, vol. 78, pp. 753-767, Apr. 1990.

[15] J.-F. Cardoso, "Super-symmetric decomposition of the fourth-order cumulant tensor. Blind identification of more sources than sensors," in Proc. IEEE ICASSP, (Toronto), pp. 3109-3112 vol.5, 1991.

[16] J.-F. Cardoso, "Iterative techniques for blind source separation using only fourth-order cumulants," in Signal Processing VI: Proc. EUSIPCO-92 (J. Vandewalle e.a., ed.), (Brussels), pp. 739-742 vol.2, Elsevier, 1992.

[17] J.F. Cardoso and A. Souloumiac, "Blind beamforming for non-Gaussian signals," IEE Proceedings F (Radar and Signal Processing), vol. 140, pp. 362-370, Dec. 1993.

[18] L. Tong, Y. Inouye, and R.-W. Liu, "Waveform-preserving blind estimation of multiple independent sources," IEEE Trans. Signal Processing, vol. 41, pp. 2461-2470, July 1993.

[19] S. Mayrargue, "A blind spatio-temporal equalizer for a radio-mobile channel using the constant modulus algorithm (CMA)," in Proc. IEEE ICASSP, pp. IV:317-320, 1994.

[20] L. Tong, G. Xu, and T. Kailath, "Blind identification and equalization based on second-order statistics: A time domain approach," IEEE Trans. Informat. Th., vol. 40, pp. 340-349, Mar. 1994.

[21] E. Moulines, P. Duhamel, J.F. Cardoso, and S. Mayrargue, "Subspace methods for the blind identification of multichannel FIR filters," in Proc. IEEE ICASSP, pp. IV:573-576, 1994.

[22] D. Slock, "Blind fractionally-spaced equalization, perfect-reconstruction filter banks and multichannel linear prediction," in Proc. IEEE ICASSP, pp. IV:585-588, 1994. 
[23] Y. Li and Z. Ding, "Global convergence of fractionally spaced Godard equalizers," in 28-th Asilomar Conf. Signals, Syst. Comp., pp. 617-621, IEEE, 1994.

[24] I. Fijalkow, F. Lopez de Victoria, and C.R. Johnson Jr., "Adaptive fractionally spaced blind equalization," in Proc. 6-th IEEE DSP Workshop, (Yosemite), pp. 257-260, IEEE, 1994.

[25] H. Liu and G. Xu, "A deterministic approach to blind symbol estimation," IEEE Signal Processing Letters, vol. 1, pp. 205-207, Dec. 1994.

[26] J.J. Shynk and R.P. Gooch, "Convergence properties of the Multistage CMA adaptive beamformer," in 27-th Asilomar Conf. Signals, Syst. Comp., pp. 622-626 vol.1, IEEE, 1993.

[27] A.V. Keerthi, A. Mathur, and J.J. Shynk, "Direction-finding performance of the multistage CMA array," in 28-th Asilomar Conf. Signals, Syst. Comp., pp. 847-852 vol.2, IEEE, 1994.

[28] B.G. Agee, "Fast adaptive polarization control using the least-squares constant modulus algorithm," in 20-th Asilomar Conf. Signals, Syst. Comp., pp. 590-595, IEEE, 1987.

[29] B.G. Agee, "Blind separation and capture of communication signals using a multitarget constant modulus beamformer," in Proc. IEEE MILCOM, (Boston), pp. 340-346 vol.2, IEEE, 1989.

[30] F. McCarthy, "Multiple signal direction-finding and interference reduction techniques," in Wescon/93 Conference Record, (San Francisco, CA), pp. 354-361, Sept. 1993.

[31] F. McCarthy, "Demonstration." Workshop on Smart Antennas in Wireless Mobile Communications, Stanford University, June 1994.

[32] B.G. Agee, "Convergent behavior of modulus-restoring adaptive arrays in Gaussian interference environments," in 22-nd Asilomar Conf. Signals, Syst. Comp., (Pacific Grove, CA, USA, Nov. 1988), pp. 818-822, IEEE, 1988.

[33] C.R. Johnson, "Admissibility in blind adaptive channel equalization," IEEE Control Systems Mag., vol. 11, pp. 3-15, Jan. 1991.

[34] H. Jamali and S.L. Wood, "Error surface analysis for the complex constant modulus adaptive algorithm," in 24-th Asilomar Conf. Signals, Syst. Comp., pp. 248-252 vol.1, Maple Press, 1990.

[35] H. Jamali, S.L. Wood, and R. Cristi, "Experimental validation of the Kronecker product Godard blind adaptive algorithms," in 26-th Asilomar Conf. Signals, Syst. Comp., pp. 1-5 vol.1, IEEE, 1992.

[36] K. Dogancay and R.A. Kennedy, "A globally admissible off-line modulus restoral algorithm for low-order adaptive channel equalisers," in Proc. IEEE ICASSP, (Adelaide), pp. III/61-64 vol.3, 1994.

[37] Y. Wang, Y.C. Pati, Y.M. Cho, A. Paulraj, and T. Kailath, "A matrix factorization approach to signal copy of constant modulus signals arriving at an antenna array," in Proc. 28-th Conf. on Informat. Sciences and Systems, (Princeton, NJ), Mar. 1994.

[38] R.W. Gerchberg and W.O. Saxton, "A practical algorithm for the determination of phase from image and diffraction plane pictures," Optik, vol. 35, pp. 237-246, 1972.

[39] B.G. Agee, "The least-squares CMA: A new technique for rapid correction of constant modulus signals," in Proc. IEEE ICASSP, (Tokyo), pp. 953956, 1986.

[40] G. Golub and C.F. Van Loan, Matrix Computations. The Johns Hopkins University Press, 1989.

[41] M.D. Zoltowski and D. Stavrinides, "Sensor array signal processing via a Procrustus rotations based eigenanalysis of the ESPRIT data pencil," IEEE Trans. Acoust., Speech, Signal Processing, vol. 37, pp. 832-861, June 1989.

[42] A.A. Shah and D.W. Tufts, "Determination of the dimension of a signal subspace from short data records," IEEE Trans. Signal Processing, vol. 42, pp. 2531-2535, Sept. 1994.

[43] L. Tong, Y. Inouye, and Liu R., "A finite-step global convergence algorithm for the cumulant-based parameter estimation of multichannel moving average processes," in Proc. IEEE ICASSP, pp. V:3445-3448, 1991.

[44] A. Bunse-Gerstner, R. Byers, and V. Mehrmann, "Numerical methods for simultaneous diagonalization," SIAM J. Matrix Anal. Appl., vol. 4, pp. 927-949, 1993

[45] M.T. Chu, "A continuous Jacobi-like approach to the simultaneous reduction of real matrices," Lin. Alg. Appl., vol. 147, pp. 75-96, 1991.

[46] P.J. Eberlein, "On the Schur decomposition of a matrix for parallel computation," IEEE Trans. Comp., vol. C-36, pp. 167-174, 1987.

[47] B.D. Flury and B.E. Neuenschwander, "Simultaneous diagonalization algorithms with applications in multivariate statistics," in Approximation and Computation (R.V.M. Zahar, ed.), pp. 179-205, Basel: Birkhäuser, 1995.

[48] A.J. van der Veen, "A Schur method for low-rank matrix approximation," SIAM J. Matrix Anal. Appl., Jan. 1996.

[49] G.W. Stewart, "An updating algorithm for subspace tracking," IEEE Trans. Signal Processing, vol. 40, pp. 1535-1541, June 1992.

[50] B. Yang, "Projection approximation subspace tracking," IEEE Trans. Signal Processing, vol. 43, pp. 95-107, Jan. 1995.
[51] G. Xu and T. Kailath, "Fast signal-subspace decomposition," IEEE Trans. Acoust., Speech, Signal Processing, vol. 42, pp. 539-551, Mar. 1994.

[52] D.J. Rabideau and A.O. Steinhardt, "Fast subspace tracking," in Proc. 7-th SP Workshop on Stat. Signal \& Array Processing, (Quebec), pp. 353-356, IEEE, June 1994.

[53] A.J. van der Veen, "Updating the Schur subspace estimator," subm. IEEE Trans. Signal Processing, Sept. 1995.

[54] A. Edelman, "The distribution and moments of the smallest eigenvalue of a random matrix of Wishart type," Lin. Alg. Appl., vol. 159, pp. 55-80, 1991.

[55] J. Lundell and B. Widrow, "Application of the constant modulus adaptive beamformer to constant and non-constant modulus algorithms," in 21-th Asilomar Conf. on Signals, Systems, and Computers, pp. 432-436 vol.1, IEEE, Nov. 1987

\section{Biography of Alle-Jan van der Veen}

Alle-Jan van der Veen (S'87, M'94) was born in The Netherlands in 1966. He graduated (cum laude) from Delft University of Technology, Department of Electrical Engineering, in 1988, and received the Ph.D. degree (cum laude) from the same institute in 1993. Throughout 1994, he was a postdoctoral scholar at Stanford University, in the Scientific Computing/Computational Mathematics group and in the Information Systems Lab. At present, he is a researcher in the Signal Processing group of DIMES, Delft University of Technology. He is the recipient of a 1994 IEEE SP paper award. His research interests are in the general area of system theory applied to signal processing, in particular system identification, time-varying system theory, and in numerical methods and parallel algorithms for linear algebra problems.

\section{Biography of Arogyaswami Paulraj}

Arogyaswami Paulraj was educated at the Naval Engineering College, India and at the Indian Institute of Technology, New Delhi (Ph.D. 1973). A large part of his career todate has been spent in research laboratories in India where he supervised the development of several electronic systems. His contributions include a sonar receiver 1973-74, a surface ship sonar 1976-83, a parallel computer 1988-91, and telecommunications systems.

$\mathrm{He}$ has held visiting appointments at several universities - Indian Institute of Technology, Delhi, 1973-74, Loughborough University of Technology, U.K. 1974-75, Stanford University, CA, USA, 1983-86. His research has spanned several disciplines, emphasizing estimation theory, sensor signal processing, antenna array processing, parallel computer architectures/algorithms and communication systems. He is currently a Professor of Electrical Engineering at Stanford University working in the area of mobile communications.

$\mathrm{He}$ is the author of about 90 research papers and holds several patents. He has won a number of national awards in India for his contributions to technology development and is a Fellow of the IEEE. 